\title{
IMPUESTOS Y DEUDA PÚBLICA: UNA RELACIÓN PARADÓJICA*
}

\author{
DANIEL VELÁZQUEZ ORIHUELA* \& DIANA XÓCHITL GONZÁLEZ GÓMEZ"** \\ UNIVERSIDAD AUTÓNOMA DEL ESTADO DE HIDALGO (MÉXICO)
}

Recibido/Received/Recebido: 29/10/2014 - Aceptado/ Accepted / Aprovado: 14/03/2016

\begin{abstract}
Resumen
En este trabajo se muestra que un incremento en los impuestos orientado a disminuir la deuda puede es incrementarla en el largo plazo. Esto ocurre siempre que un aumento en los impuestos reduzca la expectativa de ahorro en un mayor monto de lo que disminuyó la deuda pública inicialmente. La causa por la cual la deuda crece es porque el incremento en el impuesto reduce la inversión y esto tiene dos efectos: (i) disminuye el nivel de producción, lo que provoca una disminución en la base gravable, es decir, en el ingreso del gobierno; (ii) aumenta la tasa de interés y con ello el costo de la deuda pasada. Ante este panorama el gobierno se ve forzado a incrementar la deuda pública para mantener el gasto constante. En este trabajo se ofrece evidencia estadística para cinco países europeos, que muestra que la deuda pública está explicada por la inversión, tal como resulta en el modelo teórico; lo cual sugiere que las políticas de austeridad implementadas en estos países para reducir la deuda, pueden ser contraproducentes.

Palabras clave: Inversión; Política fiscal; Impuestos; Deuda; Crisis; Demanda efectiva.
\end{abstract}

\section{TAXES AND PUBLIC DEBT: A PARADOXICAL RELATIONSHIP}

\begin{abstract}
This paper shows that an increase in debt aimed at reducing taxes may increase it in the long run. This occurs whenever a tax increase reduces the expectation of savings in an amount greater than public debt decreased initially. The reason why the debt grows is because the tax increase reduces investment and this has two effects: (i) decreases the production level, which causes a decrease in the tax base, i.e., meaning the government income; (ii) the interest rate increases and thus the cost of past debt. Against this background, the government is forced to increase public debt to maintain constant spending. In this paper, statistical evidence is offered for five European countries that shows that public debt is explained by the investment, as it results in the theoretical model; this suggests that the austerity policies implemented in these countries in order to reduce debt, may be counterproductive. Keywords: Investment; Fiscal policy; Taxes; Debt; Crisis; Effective demand.
\end{abstract}

* El artículo es resultado del proyecto de investigación "Desarrollo Económico: Teoría y Práctica", financiado con recursos propios. Vigente de enero 2014 a diciembre 2015.

** Doctor en Ciencias Económicas por la Universidad Autónoma Metropolitana. Profesor Investigador del Área Académica de Economía de la Universidad Autónoma del Estado de Hidalgo (México). Líder del Cuerpo Académico de Economía. Dirección postal: Circuito La Concepción Km 2.5, Col. San Juan Tilcuautla, San Agustín Tlaxiaca Hidalgo, México C.P. 42160. Teléfono: 52 (771) 7172000 Ext. 4101. Correo electrónico: danielvelazquezo@yahoo.com.mx

*** Doctora en Ciencias Económicas por la Universidad Autónoma de Baja California. Profesora Investigadora del Área Académica de Economía de la Universidad Autónoma del Estado de Hidalgo (México). Miembro del Grupo de Investigación de Economía Aplicada. Correo electrónico: diana_xgg@hotmail.com 


\title{
IMPOSTOS E DÍVIDA PÚBLICA: UMA RELAÇÃO PARADOXAL
}

\begin{abstract}
Resumo
Neste trabalho mostra-se que um incremento nos impostos orientados a diminuir a dívida pode incrementá-la a longo prazo. Isto ocorre sempre que um aumento nos impostos reduza a expectativa de poupança em um maior montante do que diminuiu a dívida pública inicialmente. A causa pela qual a dívida cresce é porque o incremento no imposto reduz o investimento $e$ isto tem dois efeitos: (i) diminui o nível de produção, o que provoca uma diminuição na base tributável , isto é, na receita do governo; (ii) aumenta a taxa de juros e com isso o custo da dívida passada. Frente a este panorama o governo se vê forçado a incrementar a dívida pública para manter a despesa constante. Neste trabalho oferece-se evidência estatística para cinco países europeus que mostra que a dívida pública está explicada pelo investimento, tal como resulta no modelo teórico, o que sugere que as políticas de austeridade implementadas nestes países para reduzir a dívida, podem ser contraproducentes.
\end{abstract}

Palauras chave: Investimento; Política fiscal; Impostos; Dívida; Crise; Demanda efetiva.

Velázquez, D. \& González, D. (2016). Impuestos y deuda pública: una relación paradójica. En: Revista de la Facultad de Ciencias Económica: Investigación y Reflexión. rev.fac.cienc.econ, XXIV (2), DOI: http://dx.doi.org/10.18359/rfce.2223

JEL: E32, E12, H20, H63.

\section{Introducción}

El Banco Central Europeo (BCE), el Fondo Monetario Internacional (FMI) y la Comisión Europea (CE) han presionado fuertemente a España, Italia, Portugal, Irlanda y Grecia a implementar políticas fiscales de austeridad con el fin de pagar su deuda pública y así generar las condiciones de confianza, que de acuerdo a ellos, el sector privado requiere para invertir y retornar a la senda del crecimiento. Estas políticas han sido fuertemente criticadas por académicos (Krugman, 2012; Stiglitz, 2010; Díaz, 2015; Luna \& Montes de Oca, 2015; Rodríguez \& Venegas, 2015) al considerarlas ineficientes, contraproducentes y costosas en términos sociales. Este debate político es reflejo de la falta de consenso en la teoría económica sobre los efectos que causan las políticas de austeridad en la economía ${ }^{1}$.

El debate sobre los efectos de la austeridad también se ha dado en el ámbito empírico, al respecto la "hipótesis de austeridad expansiva" sostiene que la consolidación fiscal promueve el crecimiento, por lo que las políticas de austeridad
Las políticas de austeridad que han implementado las economías en crisis han sido diversas, una de ellas es el incremento en los impuestos. En la ciencia económica no hay un consenso sobre cuál es el efecto que tiene un incremento en los impuestos sobre el funcionamiento de las economías.

pueden ser expansivas (Alesina \& Perotti, 1995; Alesina \& Ardagna, 2010). En contraste, Guajardo, Leigh \& Pescatori (2014) argumentan que el método que usualmente se usa en esta literatura está sesgado para validar la hipótesis de austeridad expansiva; y muestran que la consolidación fiscal reduce la demanda privada y el nivel de producción. Blanchard \& Leigh (2013) argumentan que las políticas de austeridad aplicadas en las economías avanzadas en la actual crisis financiera han reducido el crecimiento en un monto mayor a lo esperado, y argumenta que esto puede deberse a que subestimaron el tamaño de los multiplicadores fiscales. Por otro lado, Reinhart \& Rogoff (2010) sostienen que si la deuda pública sobrepasa el 90\% del ingreso nacional, el crecimiento de la economía se reducirá drásticamente. No obstante, Herndon, Ash \& Pollin (2014) argumentan que las estimaciones realizadas por Reinhart \& Rogoff (2010) tienen serios errores metodológicos y de exclusión de datos importantes, una vez corregidos estos errores resulta que una deuda pública superior al 90\% del PIB no reduce drásticamente el crecimiento. 
En el modelo keynesiano, siempre que se considere la curva de oferta agregada horizontal, un incremento en los impuestos destinado a reducir la deuda pública disminuirá el consumo privado y con ello la demanda agregada, el empleo y la producción. En contraste, en el modelo clásico, que asume una curva de oferta agregada vertical, una mayor tasa impositiva reduce el consumo de los agentes e inicialmente la demanda agregada, sin embargo, debido a que los precios y salarios se asumen flexibles, el empleo no cambia. Así la menor demanda ocasionará una disminución en el nivel general de precios, lo cual aumentará la oferta monetaria en términos reales reduciendo la tasa de interés e incrementando así la inversión, es decir, habrá un efecto desplazamiento entre menor consumo y mayor inversión, manteniendo la demanda agregada en el nivel previo al incremento del impuesto. Pese a las diferencias en ambos modelos, en ellos se llega a la conclusión de que la deuda pública disminuirá. Una interesante revisión de estos enfoques se encuentra en Lasa (1997).

En la equivalencia ricardiana, propuesta inicialmente por Barro (1989), se argumenta que un incremento en los impuestos destinado a pagar la deuda pública no tendría por qué tener efecto alguno en la economía. La razón de esto es que cuando el gobierno incurrió en déficit, los agentes tomaron la precaución de ahorrar todos sus ingresos extras previendo que tarde o temprano el gobierno incrementaría los impuestos para pagar la deuda pública. Por lo que, el pago de la deuda pública se puede realizar sin que sea socialmente costoso.

Baxter \& King (1993) muestran, en un modelo de equilibrio general dinámico, que un incremento en los impuestos reduce los ingresos de los consumidores, lo cual los motiva a incrementar su oferta de trabajo para suavizar su consumo, a este proceso se le conoce como efecto riqueza. Linnemann \& Schabert (2003), argumentan que el efecto riqueza está presente aún si se asume mark up, rigideces en precios y monopolios, por lo que, este efecto está presente en los modelos propios de la Nueva Síntesis Neoclásica (NSN). En los modelos de oferta y demanda agregada de los nuevos keynesianos el análisis de la política fiscal se plantea en términos de cómo afecta a la brecha entre el producto y su nivel natural. Al respecto Benigno (2015) consideran que un incremento en el corto plazo del impuesto al ingreso es contractivo; sin embargo, reduce la brecha ente el producto y su nivel natural debido a que la disminución del producto es menor que la del producto natural. En cambio, un incremento en el corto plazo del impuesto al consumo reduce el producto en un mayor monto que su nivel natural, por lo que suele ampliar la brecha.

Los modelos de la NSN superan muchas de las limitaciones propias del esquema clásico y keynesiano, debido a que, a diferencia de los dos últimos, aquellos son dinámicos y de equilibrio general. Sin embargo, en estos esquemas, usualmente, el nivel de empleo lo determinan los consumidores, por lo que, el desempleo es voluntario. Siendo esto un enorme límite de la NSN, pues tras la actual crisis económica quién podría argumentar que el desempleo es voluntario.

En este artículo se propone un modelo teórico que pretende superar los límites metodológicos propios del esquema analítico clásico, keynesiano y de la NSN, es decir, es un modelo que a diferencia de los primeros dos esquemas, pero de manera análoga a la NSN, es de equilibrio general dinámico. El modelo propuesto se distingue de la NSN, al considerar el desempleo como involuntario.

El esquema que se propone es una extensión de los presentados en Velázquez (2014, 2015). En estos, a diferencia de la NSN, no está presente el modelo de Ciclo Real, por lo que las variaciones en el empleo no están determinadas por la oferta de trabajo. Con la finalidad de que el desempleo a estudiar sea involuntario se recurre al marco analítico de la Teoría de la Inexistencia del Mercado de Trabajo (TIMT), dicha teoría en su modelo básico, propuesto por Noriega (2001), se muestra que el equilibrio general competitivo es compatible tanto con el pleno empleo como con el desempleo involuntario. Por esta razón, esta teoría permite analizar la dinámica de las economías en presencia de desempleo involuntario.

En Velázquez (2015) se analiza cómo afecta un incremento del gasto público a los precios y asignaciones ofreciendo condiciones necesarias y suficientes para 
que el mayor gasto público provoque que la economía se sitúe en una trayectoria de crecimiento o decrecimiento. Velázquez (2014) estudia los efectos de un mayor impuesto sobre las asignaciones. Sin embargo, en ambos trabajos se asume que la deuda es constante a partir del segundo período, por lo que no se analiza la posibilidad de que esta crezca o no. En este artículo, a diferencia de Velázquez (2014, 2015), se asume que la deuda puede variar y se estudia cómo lo hace ante un incremento en los impuestos, además se ofrece evidencia estadística que apoye los principales resultados del modelo.

El principal resultado de nuestro modelo teórico es que un incremento en el impuesto, orientado a disminuir la deuda pública, puede en el largo plazo ser contraproducente, es decir, incrementar la misma. Esto ocurrirá siempre que disminuya la expectativa de ahorro en un mayor monto de lo que se redujo la deuda pública, en un primer momento. La razón por la cual crece la deuda pública es porque el mayor impuesto reduce la inversión lo cual tiene dos efectos: i) disminuye el nivel de producción y, con ello, la base gravable, es decir, el ingreso del gobierno, ii) incrementa la tasa de interés y, por tanto, el costo de la deuda pública. Por lo que el gobierno se ve obligado a incrementar su deuda pública para mantener constante su gasto. Este resultado es claramente contradictorio con el ofrecido por el modelo clásico keynesiano y la equivalencia ricardiana.

Nosotros ofrecemos evidencia estadística para Grecia, Italia, España, Portugal e Irlanda que muestra que el crecimiento de su deuda pública está explicado por la caída en su inversión privada, lo cual es plenamente coincidente con los resultados del modelo teórico. Por lo que, existen elementos que sugieren que las políticas de austeridad adoptadas por estos países no tendrán éxito en reducir su deuda pública si no permiten el crecimiento de la inversión ${ }^{2}$.

2 Los resultados mostrados en este artículo contribuyen a explicar por qué las políticas de austeridad no han tenido éxito en las economías europeas; sin embargo, reconocemos que es necesario analizar el papel que ha desempeñado la deuda privada. La razón de esto es que los problemas de deuda pública en los países europeos en crisis (con excepción de Grecia y en menor medida Italia y Portugal) surge a partir
El artículo está dividido en tres grandes apartados, modelo teórico, evidencia empírica y conclusiones. El modelo teórico está integrado por cinco temas: el primero se refiere a las condiciones iniciales del modelo, el segundo aborda el equilibrio general en el estado estacionario, en este se muestra que el equilibrio puede ser compatible tanto con el desempleo involuntario como con el pleno empleo. En el tercero se explica la dinámica del modelo, en el cuarto se plantea el escenario deuda-recesión, y en el último se estudia la recesión y deuda pública. La evidencia empírica se divide en metodología y resultados. Por último, se ofrecen las conclusiones.

\section{Modelo teórico}

\subsection{Condiciones iniciales del modelo}

Sea una economía en la cual conviven: un gobierno, dos consumidores (uno viejo y otro joven) y una empresa. Los tres últimos son tomadores de precios, racionales y deciden de forma descentralizada. Dado que los agentes son racionales sus expectativas también lo son. El gobierno decide sobre su consumo e ingreso de manera discrecional. La población no crece, sin embargo en todo momento hay dos consumidores, uno nacido en el período vigente (joven) y otro nacido en el periodo inmediato anterior (viejo). En este modelo no hay moneda ni bancos, por lo que para hacer posibles todas y cada una de las transacciones se recurre a la agencia central de compensaciones postulada por Debreu (1973).

Los consumidores tienen el mismo conjunto de gustos y preferencias, lo cual nos permite trabajar con un consumidor representativo. Se asume que la función de utilidad del consumidor representativo es: $U=C_{1, t}^{\alpha} C_{2, t+1}^{1-\alpha}$, donde: $0<\alpha<1$, c es el consumo,

\footnotetext{
de los rescates bancarios que convirtieron la deuda privada en pública (Díaz, 2015).Por otro lado, Heider \& Ljungqvist (2012), analizando empresas norteamericanas, muestran que la política tributaria tiene efectos asimétricos sobre la deuda privada, ya que un mayor impuesto tiende a incrementar la deuda; no obstante, la reducción de éste no disminuye el endeudamiento. Esta problemática no es discutida en este documento, pero forma parte de nuestra agenda de investigación.
} 
los subíndices 1 y 2 indican el período de vida del consumidor, $t+j \forall j=0,1,2, \ldots n$ aparecerá en todas las variables y hace referencia al período en que se realiza dicha variable. La oferta de trabajo de los consumidores es inelástica, es decir, que sin importar el vector de precios siempre se ofrece la misma cantidad ${ }^{3}$.

El consumidor en su primer período de vida financia su consumo, ahorro y el pago de impuestos con la remuneración de su trabajo y la ganancia que la empresa le otorga, por ser dueño de los derechos de propiedad de esta. El consumidor únicamente paga impuestos en su primer período de vida. En su segundo período de vida financia su consumo con su ahorro pasado más la rentabilidad de éste. Se asume que, en todo momento, las empresas son propiedad de los consumidores jóvenes ${ }^{4}$.

Las restricciones presupuestales del consumidor representativo nacido en el período $t$ son: $\Pi_{t}+w_{t} 1_{0}$ $=(1+\lambda) C_{1, t}+s_{t}$ y $\left(1+r_{t+1}\right) S_{t+1}=C_{2, t+1}$. Donde $\Pi$ es la ganancia expresada en términos reales, $w$ es el salario real, $v_{0}$ es la oferta de trabajo, la cual se considera exógena y constante a lo largo del tiempo, $\lambda$ es la tasa impositiva al consumo, $s$ es el ahorro, $\left(1+r_{t+1}\right)$ es el factor real de interés, que en ausencia de dinero y bancos equivale al precio relativo del producto, es decir, $\frac{P_{t+1}}{P}$. Por lo que, la tasa de interés se define como $r_{t+1}^{t}=\frac{P_{t+1}}{P_{t}}-1$.

De la conducta racional del consumidor nacido en el período se obtienen sus planes de demanda en

3 Esta hipótesis contrasta fuertemente con lo que usualmente se postula en los modelos de ciclo real, en estos las variaciones en los niveles de producción y empleo se suelen explicar por cambios en la oferta de trabajo, por lo que esta es altamente elástica. Lo anterior implica que el desempleo es voluntario y las reducciones en la producción son una respuesta óptima de los agentes (Baxter \& King, 1993). No obstante, debido a lo poco plausible de estos resultados en este trabajo se postula una oferta de trabajo inelástica.

4 La forma en que se transfieren los derechos de propiedad de una generación a otra se asumen exógena y sin costos, sin embargo esta hipótesis no implica que los consumidores sean altruistas. La utilidad de esta hipótesis reside en que permite que las ganancias puedan ser utilizadas para financiar la inversión. el primer y segundo período de vida y su plan de ahorro, los cuales son respectivamente:

$C_{1, t}=\alpha\left(\frac{1}{1+\lambda}\right)\left(\Pi_{t}+w_{t} 1_{0}\right)$

$C_{2, t+1}=(1-\alpha)\left(1+r_{t+1}\right)\left(\Pi_{t}+w_{t} 1_{0}\right)$

$A_{t+j}=(1-\alpha)\left(\left(\Pi_{t}+w_{t} 1_{0}\right)\right)$

Adviértase que el impuesto al consumo sólo afecta al plan de consumo del agente en su primer período de vida. Por otro lado, como es usual cuando se asume una función de utilidad tipo Cobb-Douglas, el plan de ahorro no depende de la tasa de interés.

A diferencia de como generalmente se formaliza el axioma de racionalidad en la teoría del producto, en la TIMT se asume que el productor maximiza la tasa de beneficio ${ }^{5}$ sujeto a su restricción tecnológica (Noriega, 1994). En este artículo se asumirá esta hipótesis, por lo que la conducta racional del productor se formaliza mediante el siguiente ejercicio de maximización:

$\operatorname{máx}\left(1+\Pi_{t}\right)=\frac{y_{t}}{w_{t} l_{t}+\left(1+r_{t}\right) k_{t}}$

s.a $y_{t}=\left(l_{t}-l_{t}\right)^{\beta} k_{t}$, donde: $\beta, \gamma \in \mathbb{R}^{+} \beta+\gamma<1$

En las ecuaciones (4) y (5), $\pi$ es la tasa de beneficio, es la oferta de producto, $l$ es la demanda de trabajo, $k$ es el capital, $l^{*}$ son los costos de organización.
5 La idea de que las empresas maximizan la tasa y no la masa de ganancia no es nueva, para los clásicos y Marx la variable que guía las decisiones de los capitalistas es la tasa de ganancia. Por otra parte, en Noriega (2001) y Velázquez (2013b) se analiza la racionalidad de que las empresas maximicen la tasa de ganancia, estos autores muestra que si hay dos empresas una maximizadora de la tasa y otra de la masa de ganancia y ambas demandan la misma cantidad de insumos y se enfrentan al mismo vector de precios, entonces siempre que se les permita desagregar su producción en tantas unidades productivas como sea necesario, la empresa maximizadora de la tasa de ganancia tendrá un mayor monto de ganancia que la empresa maximizadora de la masa de ganancia. Por lo que, sería más racional maximizar la tasa de ganancia que la masa. 
Los costos de organización son el trabajo que se emplea para organizar la producción, por lo que su uso no está asociado a producto positivo, es decir, sólo después de que el trabajo cubre la organización $\left(l>l^{*}\right)$ es que éste está asociado a producto positivo. Para la empresa competitiva los costos de organización son un dato. No obstante, éstos son un resultado del mercado, por lo que su magnitud es determinada en equilibrio general, y pueden variar si cambian los parámetros que determinan el equilibrio. Es válido adelantar que en el equilibrio general se obtiene que los costos de organización dependen positivamente de la demanda de producto, en consecuencia, entre mayor es la demanda que enfrenta la empresa, mayor es la organización que esta requiere para producir.

De la conducta racional del productor se obtienen sus demandas de trabajo y capital y su oferta de producto en el período t:

$$
\begin{aligned}
& l_{t}=\frac{(1-\gamma)}{(1-\beta-\gamma)} l_{t}^{*} \\
& k_{t}=\left(\frac{\gamma}{(1-\beta-\gamma)}\right) \frac{w_{t}}{\left(1+r_{t}\right)} l_{t}^{*} \\
& y_{t}=\frac{\beta^{\beta} \gamma^{\gamma}}{(1-\beta-\gamma)^{\beta+\gamma}}\left(\frac{w_{t}}{1+r_{t}}\right)^{\gamma} l_{t}^{* \beta+\gamma}
\end{aligned}
$$

La ecuación (6) es la demanda de trabajo, a diferencia de lo que usualmente se postula en la teoría neoclásica, pero de manera análoga a los resultados obtenidos en Noriega (2001) y Velázquez (2013a), la demanda de trabajo no depende del salario real, esta está determinada por los costos de organización. Más adelante se mostrará que debido a que los costos de organización tienen una relación directa con la demanda efectiva, es esta última quién determina la demanda de trabajo. Esto se debe a que las empresas ajustan su producción a la demanda efectiva vigente, así cuando ésta aumenta, las empresas se ven motivadas a contratar más trabajo para ajustar su producción a la alza.

La ecuación (7) es la demanda de capital, la cual es función positiva de los salarios y los costos de organización y negativa de la tasa de interés. La ecuación (8) es la oferta de producto, al igual que la demanda de capital, esta es función positiva del salario y de los costos de organización y negativa de la tasa de interés.

El gobierno financia su consumo presente y el pago de su deuda con un impuesto al consumo del joven y con préstamos que le otorgan los consumidores. Por lo que, su restricción presupuestal es:

$b_{t-1}\left(1+r_{t}\right)+g_{t}=\lambda c_{1, t}+b_{t}$, donde $b$ es la deuda del gobierno y $g$ es el gasto del gobierno.

Usualmente en los modelos de equilibrio general todas las transacciones ocurren en el mismo momento, lo cual permite solucionar las asignaciones de mercado de manera simultánea. No obstante, esto no es necesariamente una virtud. Robinson (1980) critica esta postura argumentando que el tiempo es histórico, por lo que los eventos se suceden unos a otros, es decir, hay una secuencia temporal explícita. Haciendo nuestra la crítica de Robinson sobre el manejo del tiempo en la teoría económica, y a diferencia de los modelos de equilibrio general, en este esquema analítico no todas las transacciones ocurren en el mismo momento. Para analizar la sucesión de eventos se postulan las siguientes hipótesis:

a) Hipótesis sobre el tiempo: Un período productivo es un conjunto no vacío conformado por una sucesión finita de momentos. El período productivo $t+j$ para todo $j=-n,-n+1,-n+2$, $\ldots 0,1,2,3, \ldots, n$ se define de la siguiente manera:

Sea $t+j \in\left\lfloor a_{t+j}, a_{t+j+1}\right\rfloor \mathrm{y}\left\lfloor a_{t+j}, a_{t+j+1}\right\rfloor \in t+j$, donde $\left|a_{t+j}-a_{t+j+1}\right| \delta$ siendo $\delta>0$ y $a_{t+j+1} \neq 0$ y $t+j \cap t+j+1=$ $\left\{a_{t+j+1} \mid a_{t+j+1} \in t+j\right.$ y $\left.a_{t+j+1} \in t+j+1\right\}$

Donde por definición $a_{t+j}$, para todo $j=-n,-n+1$, $n+2, \ldots, 0,1,2,3, \ldots, n$, se llamarán momentos. La hipótesis sobre el tiempo se representa la Ilustración 1. 
Ilustración 1. Hipótesis sobre el tiempo 6

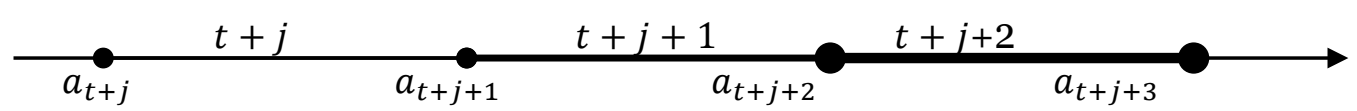

Donde:

$t+j$ está dibujado por la línea

$t+j+1$ está dibujado por la línea

b) Hipótesis sobre la sucesión de eventos:

- En el momento $a_{t+j}$ los agentes toman su deuda. Las empresas adquieren deuda para financiar su inversión del período $t+j$, y el gobierno para financiar sus egresos. Por lo que, es en este momento cuando las empresas deciden cuánto invertir. Una vez tomada la decisión de inversión las empresas no pueden retractarse. De igual forma una vez que el gobierno ha adquirido su deuda no puede anularla, pero si le es posible modificar la forma en que la utiliza, es decir, para pagar su gasto o su deuda pasada.

- En el período $t+j$, la producción comienza en $a_{t+j}+\varepsilon$, para algún $\varepsilon>0$, tal que $a_{t+j}+\varepsilon \in\left(a_{t+j}+\right.$ $\left.a_{t+j+1}\right)$.

- La decisión de cuánto trabajo demandar se toma cuando inicia la producción.

- En $a_{t+j+1}$ la producción, correspondiente al período $t+j$, se finaliza y se vende, además se pagan las deudas contraídas en el periodo pasado y los impuestos propios de este periodo.

Además de las hipótesis del tiempo y sucesión de eventos, se asumen tres hipótesis:

c) Hipótesis de miopía:

- Se asume que los consumidores y productores suelen modificar sus planes de compra y venta cuando eventos futuros en su propio período están por ocurrir, pero no son capaces de ver eventos futuros que tendrán lugar en períodos posteriores, por lo cual no modifican sus planes ante estos. Es decir, para una persona que está en el momento $a_{t+j} \in t+j$ modificará sus decisiones de compra y venta por eventos que espera que ocurran en $a_{t+j+\theta}$ siempre y cuando $a_{t+j+\theta}$ $\in t+j$. Pero no modificarán sus decisiones de compra y venta por eventos que pueden ocurrir en $a_{t+j+\theta} \in t+j+i$ donde $i=1,2, \ldots \mathrm{n}$ debido a que son agentes de vista corta.

- Los consumidores y productores son lo suficientemente pequeños_para no percibir cómo sus decisiones modifican los planes de compra y venta de todos los demás, en contraste el gobierno es lo suficientemente grande para que todos los agentes sepan cómo cambian sus planes ante modificaciones en los planes del gobierno.

- No recursividad en las expectativas. Las expectativas de los agentes siguen el orden postulado en la hipótesis de sucesión de eventos y estas no son recursivas.

Las cinco hipótesis presentadas son irrelevantes para estudiar el estado estacionario, pero son fundamentales para analizar la dinámica de la economía. El estudio inicia en estado estacionario, sin que esto signifique un rechazo a la crítica de Robinson (1980), simplemente se reconoce que para analizar la dinámica conviene partir de un punto fijo.

En el estudio del estado estacionario se resalta la compatibilidad del equilibrio tanto con el pleno empleo como con el desempleo involuntario, este resultado no depende de la intervención del Estado en la economía, pues se verifica en modelos sin gobierno (Velázquez, 2013a), pero abre la puerta

6 Fuente: Elaboración propia. 
para analizar aumentos en los niveles de empleo, que bajo la hipótesis de oferta de trabajo inelástica, sólo puede ocurrir si hay desempleo involuntario, un poco más adelante se analizará cómo cambian los niveles de empleo ante un incremento en los impuestos.

\subsection{El equilibrio general en el estado estacionario}

El principal resultado de la TIMT es demostrar que el equilibrio competitivo es plenamente compatible tanto con el pleno empleo como con el desempleo involuntario (Noriega, 2001; Rodríguez, 2005). En la TIMT, el equilibrio general se define como el vector de precios y asignaciones que, dado el salario, hacen mutuamente compatibles los planes de compra y venta realizables de los consumidores con los planes de compra y venta de los productores.

Esta definición de equilibrio general difiere de la que habitualmente se postula en la teoría neoclásica en dos aspectos: i) el salario es determinado fuera del sistema de mercados (este resultado se abordará con todo detalle un poco más adelante) ii) se trata de la compatibilidad de planes realizables. Se entiende por planes realizables, los planes de compra $y$ venta que los consumidores pueden financiar a través de sus ingresos no salariales y con la parte de su oferta de trabajo que logran que se emplee y remunere por las empresas. Los planes realizables de los consumidores son:

$$
\begin{aligned}
& c_{1, t}^{r}=\alpha\left(\frac{1}{1+\lambda}\right)\left(\Pi_{t}+w_{t} l_{t}\right) \\
& c_{2, t+1}^{r}=(1-\alpha)\left(1+r_{t+1}\right)\left(\Pi_{t}+w_{t} l_{t}\right) \\
& s_{t}^{r}=(1-\alpha)\left(\left(\Pi_{t}+w_{t} l_{t}\right)\right)
\end{aligned}
$$

El supra índice $r$ hace referencia a que se trata de planes realizables. La diferencia entre las ecuaciones (1), (2) y (3) con respecto a las ecuaciones (9), (10) y (11), es decir, la diferencia entre los planes de consumo y ahorro con respecto a sus planes realizables, es que los primeros están determinados por la oferta de trabajo, en cambio los segundos están determinados por la demanda de trabajo. Por lo que sólo en pleno empleo estos coinciden.
Se define a la demanda efectiva $\left(\hat{\mathrm{Y}}_{\mathrm{d}, \mathrm{t}}\right)$ como la suma de los planes realizables de consumo de las familias más la inversión y el gasto público realizable, es decir, $\hat{y}_{d, t}=c_{1, t}^{r}+c_{2, t+1}^{r}+k_{t+1}+g_{t}^{r}$. Por lo que, de manera análoga a los planes realizables, sólo en pleno empleo la demanda efectiva es igual a la demanda planeada.

Los planes realizables propuestos (ecuaciones 9,10 y 11) son demandas racionadas, en el entendido de que puede haber desempleo. Por lo que, incorporan la hipótesis de decisión dual planteada por Clower (1965), la cual postula que para que un agente pueda comprar tuvo que haber vendido antes, así los planes realizables de consumo y ahorro de las familias dependen del trabajo que estos hayan vendido.

De manera análoga a los equilibrios restringidos ${ }^{7}$ por demanda, en el equilibrio general propio de la TIMT las demandas nocionales ${ }^{8}$ no contienen información útil ni difunden señales de mercado, es la demanda efectiva la que determina el nivel de producción y esta depende del nivel de empleo.

Es importante aclarar que pese a las similitudes del equilibrio en la TIMT con el equilibrio restringido por demanda existe una diferencia fundamental entre estos, mientras que el último usualmente se debe a que los precios se ajustan muy lentamente (lo que da pie a que los agentes realicen transacciones a precios que no son de equilibrio), en la TIMT el desequilibrio surge porque los oferentes y demandantes de trabajo no ven en el salario real un precio que guie sus planes de compra y venta. Un poco más adelante se discutirá este punto.

Utilizando los planes realizables, en vez de las demandas nocionales, para analizar la consistencia contable del modelo se obtiene la siguiente ley de Walras modificada:

\footnotetext{
A los equilibrios restringidos también se les conoce como equilibrios no walrasianos o equilibrios con racionamiento. Para una revisión de esta literatura véase Argandoña, Gámez \& Mochón (1996).

8 Adviértase que las demandas nocionales son los planes de demanda, los cuales suelen estar en función de los ingresos planeados.
} 
$0=\left(c_{1, t}^{r}+c_{2, t}^{r}+k_{t+1}+g_{t}^{r}-y_{t}\right)+\left(1+r_{t+j}\right)\left(k_{t}+b_{t-1}^{r}-s_{t-1}^{r}\right)$

La ecuación (12) es una ley de Walras modificada, y de manera análoga a la ley de Walras habitual, esta muestra que la suma en valor de las demandas excedentes es cero. Sólo que en la TIMT una demanda excedente es la diferencia entre los planes de venta y los planes de compra realizables.

En la ley de Walras modificada sólo hay dos mercados: el de bienes en $t$ y en $t-1$; el mercado de trabajo no aparece. La razón de esto es que, en este marco analítico, el equilibrio en los mercados de bienes no implica pleno empleo, es decir, puede haber equilibrio en los mercados de bienes y desempleo, sin que esto signifique una violación a la ley de Walras modificada? .

El equilibrio en la TIMT implica que la oferta de bienes iguala a la demanda efectiva, lo cual sucede tanto en pleno empleo como en desempleo involuntario. El equilibrio se resuelve a partir de las siguientes ecuaciones:

$$
\begin{aligned}
& \hat{y}_{d, t}-y_{t}=0 \\
& k_{t}+b_{t-1}^{r}-s_{t-1}^{r}=0 \\
& l_{t}-l_{o} \leq 0
\end{aligned}
$$

La ecuación (13) es el mercado de bienes en el período $t-1$, la ecuación (14) es el mercado de bienes en el cual se garantiza siempre que el ahorro sea igual a la inversión más la deuda pública. La ecuación (15) es el mercado de trabajo el cual presumiblemente puede estar en equilibrio o no. De acuerdo con la ley de Walras modificada si el mercado de bienes en está en equilibrio, entonces el mercado de bienes en t-1 también lo estará. Así la solución del equilibrio

$9 \quad$ Es importante hacer notar que el equilibrio en los mercados de bienes en la TIMT, pueden ser vistos como desequilibrios en la ley de Walras habitual. Es decir, sigue siendo válido que bajo la noción de equilibrio propia de la tradición neoclásica, el equilibrio general es de pleno empleo, no obstante, bajo la definición de equilibrio de la TIMT el equilibrio general puede ser tanto de pleno empleo como de desempleo involuntario. Así, siempre que el desempleo se verifique puede ser visto por la tradición neoclásica como un desequilibrio. se obtiene a partir del mercado de bienes en $t$ o en $t-1$ y del mercado de trabajo.

La solución del equilibrio general se realizará en el estado estacionario, es decir, en aquella situación en la cual las variables son constantes en el tiempo, razón por la cual en esta sección se prescindirá de los sub índices temporales. La solución del equilibrio se realizará con base en las ecuaciones (13) y (15).

Sustituyendo los respectivos planes de compra y venta realizables de los agentes en los mercados de bienes (ecuación 13) y trabajo (ecuación 15), se obtiene:

$$
\begin{aligned}
& \alpha\left(\frac{\beta^{\beta} \gamma^{\gamma}}{(1-\beta-\gamma)^{\beta+\gamma}}\left(\frac{w}{1+r}\right)^{\gamma} l^{* \beta+\gamma}-\frac{\gamma}{1-\beta-\gamma} w l^{*}\right)+(1-\alpha)(1+r) \\
& \left(\frac{\beta^{\beta} \gamma^{\gamma}}{(1-\beta-\gamma)^{\beta+\gamma}}\left(\frac{w}{1+r}\right)^{\gamma} l^{* \beta+\gamma}-\frac{\gamma}{1-\beta-\gamma} w l^{*}\right)+\frac{\gamma}{1-\beta-\gamma} \frac{w}{(1+r)} l^{*}+ \\
& b-(1+r) b-\frac{\beta^{\beta} \gamma^{\gamma}}{(1-\beta-\gamma)^{\beta+\gamma}}\left(\frac{w}{1+r}\right)^{\gamma} l^{* \beta+\gamma}=0 \\
& \frac{(1-\gamma)}{1-\beta-\gamma} l^{*}-l_{o} \leq 0
\end{aligned}
$$

Las ecuaciones (16) y (17) son, respectivamente, el mercado de bienes y el de trabajo. El primero está en función de la tasa de interés, el salario y los costos de organización, el segundo está determinado sólo por los costos de organización. La razón por la cual no aparece el salario real como un elemento del mercado de trabajo es porque los oferentes y demandantes no ven en éste una variable que guie sus planes de compra y venta.

El equilibrio general en estado estacionario implica que el precio en el período $t$ sea el mismo que en el período $t-1$. Por lo que, el factor de interés es igual a la unidad $y$, por tanto, la tasa de interés es cero ${ }^{10}$, es decir: $(1+r)=1$. Cuando ello ocurre la ecuación (16) es una igualdad estricta independientemente de cuál sea el salario real y los costos de organización. Lo anterior implica que siempre que los precios sean de equilibrio, el mercado de bienes se vaciará sin importar cuál sea el salario real.

\footnotetext{
10 Adviértase que por definición $r_{t+j}=\frac{p_{t+j}-p_{t+j-1}}{p_{t+j-1}}$, por lo que $r_{t+j}=0$, si y sólo si $p_{t+j}=p_{t+j-1}$
} 
La ecuación (17) determina los costos de organización, sin embargo no hay ningún mecanismo de mercado que garantice que estos sean de pleno empleo, es decir, los costos de organización pueden ser o no de pleno empleo y, aun así, sostenerse el equilibrio en el mercado de bienes.

Adviértase que una vez que se determina el precio relativo de equilibrio $((1+r)=1)$, el salario real queda indeterminado, independientemente de si hay o no pleno empleo, lo cual implica que éste no se resuelve en ningún mercado, es decir, el salario real se determina fuera del sistema de mercados. Esto es porque los oferentes y demandantes de trabajo no ven al salario real como el precio que determine sus planes de oferta y demanda.

En la TIMT, los mercados están compuestos por tres elementos: oferentes, demandantes y un precio que vincule sus planes de compra y venta. $\mathrm{Al}$ no haber un precio que vincule los planes de compra y venta de trabajo se afirma que el mercado de trabajo no se forma. A la confluencia de oferentes y demandantes que no están vinculados por un precio se le llama sector, de ahí que se hable del sector laboral. ${ }^{11}$ Con la finalidad de ser congruentes con este marco analítico, a partir de este momento nos referiremos al mercado de trabajo como sector laboral.

El hecho de que el salario real no se determine en el sistema de mercados implica que una economía competitiva está conformada por lo menos por dos instituciones ${ }^{12}$ : el mercado y otra en donde se determina el salario real ${ }^{13}$. Para propósitos de esta investigación bastará con tratar al salario real como una variable

11 Es interesante reflexionar que en la teoría del equilibrio general competitivo se afirma que el principal resultado del mercado son los precios, al estar determinado el salario real fuera del sistema de mercados no es posible afirmar que éste sea un resultado de mercado alguno, lo cual reafirma la hipótesis de inexistencia del mercado de trabajo.

12 Se entiende por institución las reglas que son socialmente aceptadas.

13 Es decir, ninguna economía competitiva puede tener como única institución al mercado. A diferencia de la teoría neoclásica, en la TIMT, en una economía competitiva, la cohesión social no está determinada únicamente por el vector de precios, sino por el vector de precios y las convenciones sociales que determinan el salario real. exógena. No obstante, se reconoce que analizar cómo se determina esta es una de las principales líneas de investigación pendientes en este marco analítico. A partir de la ecuación (17) se puede saber si los costos de organización son o no de pleno empleo, pero no es útil para determinar los costos de organización. Para analizar cómo se determinan éstos se sustituye la ecuación (8) en (16):

$$
l^{*}=\left(\frac{(1-\beta-\gamma)^{\beta+\gamma}}{\beta^{\beta} \gamma^{\gamma}}\left(\frac{1+r}{w}\right)^{\gamma} \hat{y}_{d}\right)^{\frac{1}{\beta+\gamma}}
$$

La ecuación (18) muestra que los costos de organización tienen una relación positiva con la demanda efectiva. En Noriega (2001) se argumenta que esto es debido a que entre más grande es la demanda efectiva que enfrenta una empresa mayor será el número de transacciones que tendrá que realizar, para lo cual requerirá una mayor organización. La relación que guardan los costos de organización con la tasa de interés y con los salarios es un poco más complicada pues estos suelen modificar a la demanda efectiva. Sustituyendo los costos de organización (ecuación 18) en los planes de compra y venta del productor (ecuaciones 6,7 y 8 ) resulta:

$$
\begin{aligned}
& l=\frac{(1-\gamma)}{(1-\beta-\gamma)}\left(\frac{(1-\beta-\gamma)}{\beta^{\beta} \gamma^{\gamma}}\left(\frac{1+r}{w}\right)^{\gamma} \hat{y}_{d}\right)^{\frac{1}{\beta+\gamma}} \\
& k=\frac{\gamma}{1-\gamma} \frac{w}{(1+r)}\left(\frac{(1-\beta-\gamma)}{\beta^{\beta} \gamma^{\gamma}}\left(\frac{1+r}{w}\right)^{\gamma} \hat{y}_{d}\right)^{\frac{1}{\beta+\gamma}} \\
& y=\hat{y}_{d}
\end{aligned}
$$

La ecuación (21) muestra que la producción es determinada por la demanda efectiva. Esto se debe a que si las empresas producen menos de lo que el mercado les demanda, entonces no ganarían tanto como pueden ganar, pero si producen más, entonces generarían inventarios no deseados y, por tanto, pérdidas. Este resultado es análogo al obtenido en Keynes (1936).

La demanda de trabajo (ecuación 19) es análoga a la función ocupación ${ }^{14}$ propuesta en Keynes

\footnotetext{
14 Para Keynes (1936, p. 270) "la función ocupación estará determinada por $N_{v}=F\left(D_{s}\right)$. Lo que quiere decir que $N$, hombres estarán empleados en la industria $r$ cuando la demanda efectiva sea $D_{s}$ ".
} 
(1936), al igual que ésta, muestra que el nivel de empleo depende de forma positiva de la demanda efectiva. La ecuación (20) es la demanda de inversión, la cual también depende de manera positiva de la demanda efectiva. La razón por lo cual tanto la demanda de trabajo como la inversión dependen de la demanda efectiva se debe a que las empresas ajustan su producción a la demanda efectiva vigente; por lo que, cuando esta aumenta suelen demandar más insumos.

Adviértase que pese a la afirmación de que no existe un mercado de trabajo, el equilibrio general en la TIMT está basado en un razonamiento de multimercados, pues de acuerdo con la ley de Walras modificada (ecuación 12) lo que le ocurra al mercado de bienes en $\mathrm{t}-1$ le afecta al mercado de bienes en $t$ y viceversa. La no existencia del mercado de trabajo simplemente implica que el nivel de empleo y el salario no se pueden razonar como si fueran resultado de un mercado, es decir, ni el salario real es determinado por la interacción de oferentes y demandantes de trabajo, ni el empleo es determinado por el salario real. Estos son determinados por procesos distintos a los de mercado ${ }^{15}$.

Es importante aclarar que no es posible determinar paramétricamente las asignaciones de equilibrio, sin determinar exógenamente al salario real así como al nivel de empleo, siendo esto una consecuencia de que no se forme un mercado de trabajo, por lo que las asignaciones y los precios que de él se desprenderían no se determinan. No obstante, a partir de una situación de equilibrio sí es posible determinar cómo evolucionarían los precios y las asignaciones ante un shock exógeno.

En el siguiente apartado se analizará la dinámica del modelo, es decir, cómo cambian las asignaciones

15 En las teorías de equilibrio general razonar el nivel de empleo y el salario fuera de la lógica de mercado no es del todo novedoso, por ejemplo en la teoría de los precios propuesta por Karl Marx se razona los niveles de empleo y salario fuera de cualquier lógica de mercado, al respecto Neffa, Féliz, Panigo \& Pérez (2006, p. 54) mencionan: "Para Marx, el mercado de trabajo no existe como tal, es decir, como espacio en donde interactúan la oferta y la demanda de fuerza de trabajo, ya que los salarios y el empleo no se determinan en un mismo proceso ni dependen de los mismos factores". y los precios cuando la economía sale del estado estacionario, para ello se asumirá que el gobierno decide incrementar, sólo una vez, los impuestos y utilizar la mayor recaudación para reducir su deuda. Dado que el salario se determina fuera del sistema de mercados se asumirá, por simplicidad, que éste es constante en el tiempo y conocido por todos los agentes.

\subsection{Dinámica del modelo}

\subsubsection{El efecto de un incremento en el impuesto al consumo, en el período $t^{16}$}

De manera análoga al trabajo de Velázquez (2014), se supondrá una economía en estado estacionario y con desempleo involuntario. Al inicio del período $t$, es decir, en el momento $a_{t}$ el gobierno decide incrementar el impuesto al consumo con la finalidad de contratar menos deuda. Adviértase que el impuesto únicamente se incrementa en este período, en los períodos posteriores éste permanece constante. La reducción de la deuda pública equivale al incremento esperado en los ingresos tributarios. Siempre que el gobierno acierte en sus expectativas recaudará lo suficiente para financiar su recorte en deuda sin modificar su gasto. No obstante, si las expectativas no se verifican, el gobierno tendrá que ajustar su gasto para sujetarse a su restricción presupuestal.

En el momento $a_{t}$ todas las variables que se realizan posterior a este momento son expectativas, no obstante, por simplicidad sólo se utilizará el operador $\mathrm{E}$ en aquellas variables que se realizan en el período $t$.

De acuerdo a las hipótesis de tiempo y sucesión de eventos, en el momento $a_{t}$, los agentes están decidiendo su inversión con base en la tasa de interés. Por lo que, se iniciará analizando la forma en la que el incremento en el impuesto al consumo afecta a la tasa de interés. De acuerdo con las ecuaciones de este modelo se tiene:

$\frac{d\left(1+r_{t+1}\right)}{d \lambda_{t}}=\frac{(1-\alpha)\left(\left(\frac{\beta}{1-\gamma}\right)^{\beta} k_{t}^{\gamma} \frac{d E\left(l_{t}^{\beta}\right)}{d \lambda_{t}}-\left(\frac{\gamma}{1-\gamma}\right) w_{t} \frac{d E\left(l_{t}\right)}{d \lambda_{t}}\right)-\frac{d b_{t}}{d \lambda_{t}}}{-\left(\frac{\gamma}{1-\gamma}\right) \frac{w_{t+1} l_{t+1}}{\left(1+r_{t+1}\right)^{2}}} \lesseqgtr 0$

16 El desarrollo matemático de la dinámica del modelo se presenta en el anexo. 
La ecuación (22) muestra que el efecto que tiene un incremento en la tasa impositiva al consumo sobre la tasa de interés es ambiguo, con base en el denominador de esta ecuación se obtiene las condiciones suficientes y necesarias para que un incremento en los impuestos aumente, reduzca o no modifique a la tasa de interés.

$$
\begin{aligned}
& (1-\alpha)\left(\left(\frac{\beta}{1-\alpha}\right)^{\beta} k_{t}^{\gamma} \frac{d E\left(l_{t}^{\beta}\right)}{d E\left(l_{t}\right)}-\left(\frac{\gamma}{1-\gamma}\right) w_{t}\right) \frac{d E\left(l_{t}\right)}{d \lambda_{t}} \lesseqgtr \frac{d b_{t}}{d \lambda_{t}} \rightarrow \\
& \frac{d\left(1+r_{t+1}\right)}{d \lambda_{t}} \gtreqless 0
\end{aligned}
$$

El primer miembro de la primera inecuación de la ecuación (23) es la reducción en el ahorro esperado ante un incremento en la tasa impositiva en el consumo, el segundo miembro es la reducción en la deuda pública. En consecuencia la ecuación (23) muestra que siempre que los agentes esperen que el ahorro se reduzca en un mayor (menor o igual) monto que la disminución en la deuda pública, entonces la tasa de interés aumentará (disminuirá o no se modificará).

Pero, ¿por qué los agentes esperan que el ahorro disminuya? Para responder esta pregunta adviértase que a lo largo de todo el documento se asumirá que el salario satisface la siguiente desigualdad:

$$
\left(\frac{\beta}{1-\alpha}\right)^{\beta} k_{t+j}^{\gamma} \frac{d E\left(l_{t+j}^{\beta}\right)}{d E\left(l_{t+j}\right)} \frac{(1-\gamma)}{\gamma}>w_{t+j}
$$

La ecuación (24) implica que la forma en quelos agentes esperan que cambie el ahorro (primer miembro de la ecuación (23) dependa de la expectativa que los agentes tengan sobre la evolución del empleo en ese período. En consecuencia, la expectativa de una reducción en los niveles de ahorro se verificará siempre que los agentes esperen que el nivel de empleo disminuya, pero ¿Por qué los agentes esperan que el empleo disminuya ante un incremento en el impuesto al consumo? Para responder esta pregunta adviértase que del modelo resulta:

$$
\begin{gathered}
\frac{d E\left(\hat{y}_{t}\right)}{d \lambda_{t}}=\frac{d E\left(c_{t}\right)}{d \lambda_{t}}<0 \\
\frac{d E\left(l_{t}\right)}{d \lambda_{t}}=\left(\frac{1-\gamma}{\beta}\right) k_{t}^{-\frac{\gamma}{\beta}} \frac{d E\left(\hat{y}_{d t}^{\frac{1}{\beta}}\right)}{d E\left(\hat{y}_{d t}\right)} \frac{d E\left(\hat{y}_{d t}\right)}{d \lambda_{t}}<0
\end{gathered}
$$

La ecuación (25) muestra que las empresas esperan que la demanda efectiva disminuya, esto debido a que el incremento en el impuesto al consumo encarece a este último, por lo que lo reduce. De hecho la reducción esperada en la demanda efectiva es proporcional a la disminución esperada en el consumo. La ecuación (26) muestra que ante la expectativa de una menor demanda efectiva, las empresas esperan contratar menos trabajo. Es importante resaltar que las empresas modifican su demanda de trabajo para ajustar su producción a la demanda efectiva vigente. La expectativa de un menor nivel de empleo implica que los agentes tendrán una disminución en sus ingresos, lo cual reducirá su capacidad de ahorro. Esto explica por qué los agentes esperan que el ahorro disminuya ante un incremento en el impuesto al consumo.

Adviértase que el incremento de la tasa impositiva al consumo desencadena dos fuerzas contrarias sobre la tasa de interés, provoca que los agentes tengan la expectativa de un menor ahorro, por el otro lado disminuye la deuda pública. Por lo que, la tasa de interés se ajusta para garantizar la igualdad ahorroinversión-deuda.

El cambio en la tasa de interés modifica las asignaciones en este período, con la finalidad de analizar cómo cambian estas analizaremos las ecuaciones que resultan del modelo:

$$
\begin{aligned}
\frac{d k_{t+1}}{d \lambda_{t}} & =-\left(\frac{\gamma}{1-\gamma}\right) \frac{w_{t+1} l_{t+1}}{\left(1+r_{t+1}\right)^{2}} \frac{d\left(1+r_{t+1}\right)}{d \lambda_{t}} \gtreqless 0 \\
\frac{d k_{t+1}}{d \lambda_{t}} & =-\left(\frac{\gamma}{1-\gamma}\right) \frac{w_{t+1} l_{t+1}}{\left(1+r_{t+1}\right)^{2}} \frac{d\left(1+r_{t+1}\right)}{d \lambda_{t}} \gtreqless 0 \frac{d E\left(l_{t}\right)}{d \lambda_{t}}<0 \\
\frac{d l_{t}}{d \lambda_{t}} & =\frac{\frac{l_{t} d \hat{y}_{d t}}{1-\frac{1 l_{t} d \lambda_{t} d t}{\beta \hat{y}_{d, t} d l_{t}}}}{\hat{y}_{1}}
\end{aligned}
$$

La ecuación (27) muestra que la decisión de inversión se modifica a partir de la forma en que cambia la tasa de interés, es decir, si la tasa de interés se reduce (aumenta, no cambia), entonces la inversión crece (decrece, no cambia). La demanda efectiva cambia debido a la disminución en el consumo esperado y a la variación en la inversión. La inecuación (28) muestra que, sin importar cómo cambie la 
inversión, la demanda efectiva disminuirá. La razón de esto es que aún en el caso de que la inversión aumente, esta lo hará en un monto menor que la reducción de la deuda pública; no obstante, el consumo disminuye en la misma proporción en que se redujo la deuda.

La ecuación (29) muestra que, ante la caída en la demanda efectiva, las empresas se ven motivadas a reducir su demanda de trabajo para ajustar su producción a la baja. Adviértase que el denominador de la ecuación (29) es positivo siempre que la elasticidad trabajo-producto sea estrictamente mayor que la elasticidad trabajo-demanda, es decir, siempre que el trabajo genere más recursos de los que consume. Esta condición se verifica si y sólo si: $\beta \frac{(1-\gamma)}{\gamma} \frac{y_{t}}{l_{t}}>w_{t}$. Se asumirá que esta desigualdad, y su expectativa, se sostienen sistemáticamente en todos los periodos.

Al final del período $t$, es decir, en el momento $a_{t+1}$ la producción se habrá reducido como consecuencia de la disminución de la demanda efectiva. En este momento el presupuesto del gobierno estará bajo la presión de dos fuerzas contrarias: la disminución en el ingreso tributario y la reducción en el costo de la deuda pasada. Por un lado la caída en la producción y el empleo implican que la base gravable habrá disminuido $y$, por tanto, los ingreso tributarios; por otro lado, la reducción en el empleo hará menos productivo al capital vigente $\mathrm{y}$, por tanto, reducirá la tasa de rentabilidad del ahorro pasado, es decir, la tasa que paga el gobierno por la deuda contraída en el período anterior. Así, de las ecuaciones del modelo resulta:

$\frac{d g_{t}}{d \lambda_{t}}=\alpha\left(\frac{\lambda_{t}}{1+\lambda_{t}}\right)\left(\beta \frac{y_{t}}{l_{t}}-\frac{\gamma}{1-\gamma} w_{t}\right) \frac{d l_{t}}{d \lambda_{t}}-\left(\frac{\gamma}{1-\gamma}\right) \frac{w_{t}}{k_{t}} b_{t-1} \frac{d l_{t}}{d \lambda_{t}} \lesseqgtr 0$

En la ecuación (30), el primer miembro del lado derecho de esta ecuación es la disminución en los ingresos tributarios, el segundo miembro es la disminución en la tasa de rentabilidad del ahorro pasado, es decir, del costo de la deuda pasada. La ecuación (30) muestra que el gasto público se ajustará para garantizar el equilibrio presupuestal del gobierno, aunque a priori no es evidente qué fuerza dominará.

Los resultados hasta aquí presentados contrastan con los obtenidos por Barro (1989), es decir, no se verifica la equivalencia ricardiana. La razón de esto es que los agentes tienen miopía, por lo que no fueron capaces de anticipar, cuando surgió la deuda pública, que esta tarde o temprano tendría que ser pagada con mayores impuestos.

A diferencia del modelo clásico, pero de manera análoga al modelo keynesiano ${ }^{17}$, la producción y el empleo se reducen a consecuencia del incremento en el impuesto. La razón de esto es porque la producción se ajusta a la demanda efectiva y un aumento en los impuestos reduce esta. A diferencia del modelo keynesiano, el modelo aquí presentado permite estudiar la trayectoria que sigue la economía en los períodos posteriores al incremento en el impuesto.

\subsubsection{Los precios y las asignaciones en los períodos posteriores a $t$}

La trayectoria que siga la economía en el largo plazo dependerá de la forma en que cambió la inversión en el período t. La razón de esto es que la decisión de inversión suele trascender en el tiempo debido a que la inversión de hoy es parte de la capacidad productiva de mañana. Al inicio del período $t+1$, es decir, en $a_{t+1}$ la tasa de interés se modifica a consecuencia del cambio en la inversión pasada, con base en las ecuaciones del modelo se tiene:

$$
\frac{d\left(1+r_{t+2}\right)}{d \lambda_{t}}=\frac{(1-\alpha) \gamma \frac{y_{t+1} d k_{t+1}}{k_{t+1} d \lambda_{t}}}{-\left(\frac{\gamma}{1-\gamma}\right) \frac{w_{t+2} l_{t+2}}{\left(1+r_{t+2}\right)^{2}}} \lesseqgtr 0
$$

Nótese que a diferencia de la manera en cómo se modificó la tasa de interés en el período pasado, en este período el cambio en la tasa de interés no depende de la expectativa sobre el nivel de empleo. La razón de esto es que el incremento en el impuesto al consumo ocurrió en el periodo pasado, por lo que en el período actual el cambio en los niveles de empleo obedece a las modificaciones en las decisiones de los agentes. Sin embargo, las familias y las empresas son tan pequeñas que no perciben cómo sus decisiones modifican a la economía. Por ello no

17 Se entiende por modelo clásico al modelo IS-ML con curva de oferta vertical, el keynesiano al modelo IS-ML con curva de oferta horizontal. 
esperan que sus decisiones individuales modifiquen los niveles de empleo. Por otro lado, el cambio en la inversión pasada es conocido por todos, por lo cual su variación es tomada en cuenta para determinar la nueva tasa de interés.

La ecuación (31) muestra que la forma en que cambia la tasa de interés depende esencialmente de lo que haya pasado con la inversión en el periodo inmediato anterior, tal que si:

$$
\frac{d k_{t+1}}{d \lambda_{t}} \lesseqgtr 0 \rightarrow \frac{d\left(1+r_{t+j+2}\right)}{d \lambda_{t}} \gtreqless 0
$$

La ecuación (32) muestra tres escenarios posibles: i) si la inversión pasada disminuyó, entonces la tasa de interés aumentará, ii) si la inversión pasada creció, entonces la tasa de interés se reducirá, iii) si la inversión pasada no cambió, entonces la tasa de interés tampoco lo hará. La razón de esto es que la menor (mayor) inversión pasada implica una disminución (aumento) en la capacidad productiva presente $\mathrm{y}$, por tanto, en las expectativas de los agentes sobre el ahorro. Bajo este mismo razonamiento si la inversión pasada no cambia, entonces tampoco lo hará la expectativa de los agentes sobre el ahorro. Estos tres escenarios conducen a distintas trayectorias de la economía en el largo plazo.

Es importante resaltar que en última instancia la razón por la cual la inversión pasada se modificó es porque el incremento en el impuesto tuvo dos efectos: i) redujo la expectativa de ahorro, es decir, destruyó recursos, ii) disminuyo la deuda pública, es decir, liberó recursos. Así el primer escenario que resulta de (32), en última instancia se debe a que el impuesto destruyó más recursos de los que liberó, el segundo a que el impuesto destruyó menos recursos de los que liberó, el tercero a que el impuesto destruyó los mismos recursos que liberó.

En Velázquez (2014) se analizan estos tres escenarios, en el primero se muestra que la economía se sitúa en una senda de recesión en la cual el gobierno se ve obligado a reducir su gasto público para no contraer más deuda, en el segundo escenario la economía se encuentra en un nuevo estado estacionario, pero a diferencia del estado estacionario ini- cial, el nivel de empleo y producción son menores. Finalmente, en el tercer escenario la economía se ubica en una senda de crecimiento en la cual puede incrementar el gasto público sin incrementar impuestos ni recurrir a déficit.

En este artículo se analiza el primer escenario, siguiendo el trabajo de Velázquez (2014), debido a que en éste se encuentran elementos que pueden explicar por qué las políticas de austeridad, adoptadas por las economías europeas en crisis, han sido incapaces de reducir su deuda. Posteriormente, a diferencia de Velázquez (2014), se asumirá que el gobierno, presionado por la caída en sus ingresos, se resiste a reducir su gasto, por lo que recurre a deuda pública para financiarlo.

\subsection{Escenario deuda-recesión}

A partir de ahora se asumirá que la inversión en el período pasado disminuyó. La menor inversión en $t$ reduce la capacidad productiva en $t+1 \mathrm{y}$, por tanto, genera una expectativa de un menor ahorro. Esta expectativa provoca que aumente la tasa de interés en $t+1$ provocando que la inversión en $t+1$ se reduzca. Así en última instancia la inversión en disminuye debido a que la inversión en $t$ se redujo como lo muestra la ecuación (33)

$$
\frac{d k_{t+2}}{d \lambda_{t}}=(1-\alpha) \gamma \frac{y_{t+1}}{k_{t+1}} \frac{d k_{t+1}}{d \lambda_{t}}<0
$$

La menor inversión tanto pasada como presente reduce la demanda efectiva. Sin embargo, debido a que la caída en la inversión presente se debe a la menor inversión pasada, la reducción en la demanda efectiva se debe en última instancia a la menor inversión pasada, como se muestra en la inecuación (34).

$$
\frac{d \hat{y}_{d, t+1}}{d \lambda_{t}}=\gamma \frac{y_{t+1}}{k_{t+1}} \frac{d k_{t+1}}{d \lambda_{t}} \lesseqgtr 0
$$

La menor demanda efectiva genera incentivos para que las empresas contraten menos trabajo. No obstante, la reducción de la inversión pasada implica que las empresas tienen una menor capacidad productiva. Por lo que, tanto la demanda efectiva como la capacidad productiva decrecieron. Si la demanda 
efectiva disminuyó más (menos) que la capacidad productiva, las empresas tendrán incentivos para reducir (aumentar) su demanda de trabajo. De este razonamiento se desprende la inecuación (35).

$$
\eta_{\lambda_{t} \hat{y}_{d, t+1}} \gtreqless \eta_{k_{t+1}, y_{t+1}} \eta_{\lambda_{t}, k_{t+1}} \rightarrow \frac{d l_{t+1}}{d \lambda_{t}} \gtreqless 0
$$

La inecuación (35) muestra que si elasticidad tasa impositiva-demanda efectiva $\left(\eta_{\lambda_{t} \hat{y}_{a}, \underline{t} s_{1}}\right)$ nenor (ma-yor o igual) al producto de las elasticidades tasa impositiva-capital y capital-producción $\left(\eta_{k_{t+1}, y_{t+1}} \eta_{\lambda_{t}, k_{t+1}}\right)$ entonces el nivel de empleo disminuirá (aumentará o no cambiará). Es decir, siempre que un aumento en el impuesto al consumo disminuya a la demanda efectiva en un mayor monto que la capacidad productiva, entonces el empleo se reducirá, en el caso contrario el empleo aumentará. La razón de esto es que las empresas maximizan su ganancia cuando producen todo lo que el mercado les demanda, por lo que ajustan su demanda de trabajo para garantizar que su producción coincida con la demanda efectiva vigente.

Existen dos puntos a resaltar en este análisis: i) la capacidad del impuesto al consumo de generar efectos que trasciendan en el tiempo, ii) la forma en que el impuesto afecta tanto a la capacidad productiva como a la demanda efectiva.

Con respecto al primero, la razón por la que los efectos de un incremento del impuesto al consumo son capaces de trascender en el tiempo se debe a que modifica la decisión de inversión de su período. Es el cambio en la inversión la que trasciende en el tiempo modificando los precios y las asignaciones en los períodos subsecuentes al aumento del impuesto.

Con respecto al segundo punto, el incremento en el impuesto modifica tanto la demanda efectiva como la capacidad productiva porque influye sobre la inversión, es decir, es el carácter dual de la inversión (capaz de generar tanto demanda efectiva como capacidad productiva) la que permite esto. Adviértase, que en el período en que se toma la decisión de inversión esta forma parte de la demanda efectiva, no obstante para el siguiente período forma parte de la capacidad productiva de la economía. Por lo que, es el cambio en la inversión la que determina el cambio en el nivel de empleo, esto se muestra en la inecuación (36).

$$
\frac{d t_{t+1}}{d \lambda}=\frac{\frac{\gamma t_{t+1}}{\beta q_{k t+1}}\left(\frac{E\left(q_{t+1}\right)}{\widehat{q}_{t+1}}-1\right) \frac{d q_{k t+1}}{d \lambda}}{1-\frac{\eta_{\lambda, t}}{\beta}} \gtreqless 0
$$

La inecuación (36) muestra que siempre que la expectativa sobre el nivel de producción sea mayor (menor) que la demanda efectiva, entonces el empleo cambiará en el mismo sentido (sentido contrario) en que lo hizo la inversión. Por lo que, de acuerdo a (35), si la expectativa del producto es mayor (menor) a la demanda efectiva, entonces esta última se habrá reducido en un mayor (menor) monto que la capacidad productiva, por lo cual las empresas demandarán menos trabajo para ajustar su producción a la menor demanda efectiva.

Nosotros mostramos evidencia empírica de cinco países de Europa, en donde se muestra que el empleo sigue la misma trayectoria que la inversión. Por lo que asumiremos que la inecuación (36) es negativa. Con base en las estadísticas de International Financial Statistics del Fondo Monetario Internacional y World Development Indicators del Banco Mundial se estimó un panel dinámico mediante el Método General de Momentos, con observaciones anuales de empleo e inversión para Grecia, España, Portugal, Irlanda e Italia en el periodo 1990 a 2015, en el programa Stata 14.1. El modelo estimado es $L_{i t}=\alpha+\beta L_{i t-1}+\gamma k_{i t-1}$. Conforme a los resultados obtenidos en la figura 1 se confirma una relación directa entre el empleo y la inversión rezagada en un periodo, cuya elasticidad es 0.046 al 99 por ciento de confianza.

La forma en que en este modelo se explican los cambios en los niveles de empleo contrasta fuertemente con la idea que habitualmente se ofrece en los modelos de equilibrio general dinámico. En el trabajo seminal de Baxter \& King (1993), un aumento en el impuesto incrementa el nivel de empleo debido a que ante el mayor impuesto los consumidores ven reducida su riqueza $\mathrm{y}$, por tanto, incrementan su oferta de trabajo, a este mecanismo usualmente, en la literatura especializada, se le llama efecto riqueza. 
Tabla 1. Estimaciones del empleo y la inversión

\begin{tabular}{|l|l|l|l|l|l|}
\hline \multicolumn{3}{|c|}{ Número de observaciones $=125$} & \multicolumn{3}{c|}{ Wald chi2(2) $=54110.30$} \\
\hline \multicolumn{3}{|c|}{ Número de grupos $=\mathbf{5}$} & \multicolumn{3}{c|}{ Prob $>$ chi2 $=\mathbf{0 . 0 0 0 0}$} \\
\hline Empleo & Coef. & Error estándar & $\mathrm{P}>|\mathrm{z}|$ & {$[95 \%$ Intervalo de confianza $]$} \\
\hline Empleo L1 & 0.9350 & 0.0072 & 0.000 & 0.9208 & 0.9491 \\
\hline Inversion L1 & 0.0461 & 0.0054 & 0.000 & 0.0354 & 0.0567 \\
\hline const & -0.3807 & 0.0466 & 0.000 & -0.2893 & -0.4720 \\
\hline
\end{tabular}

Fuente: Estimaciones propias con información del Fondo Monetario Internacional y Banco Mundial.

Una de las principales características de este efecto es que los cambios en los niveles de empleo se explican por modificaciones en la oferta de trabajo, por lo que el desempleo es voluntario. Cabe aclarar que el efecto riqueza está presente en la mayoría de los modelos de equilibrio general dinámico, incluso en aquellos que postulan monopolios y rigideces en precios, es decir, en el marco de la nueva síntesis neoclásica (NSN). Al respecto Linnemann \& Schabert (2003) demuestran que los supuestos de precios rígidos, mark up y una regla de Taylor no son suficientes para producir resultados que se desvíen mucho de los presentados por Baxter \& King (1993), es decir, los cambios en los niveles de empleo se siguen explicando con base en el efecto riqueza.

La principal diferencia que existe entre la forma en que se explica el cambio en el nivel de empleo entre el modelo aquí presentado y los modelos propios de la NSN radica en que en estos últimos el desempleo es voluntario, en contraste en este modelo el desempleo es involuntario. Pero a la luz de las altas tasas de desempleo que actualmente sufren economías como la española, italiana, griega, irlandesa y portuguesa, y el desánimo y desesperanza que esto ha generado al interior de sus sociedades ¿quién podría sostener que el desempleo es voluntario?

Por otro lado, la explicación sobre los cambios en el empleo que se ofrece es análoga al razonamiento de Harrod (1939), de acuerdo a este autor el nivel de empleo se mueve en el mismo sentido que la inversión y esta última crece cuando la demanda es mayor que la oferta y disminuía cuando ocurre lo contrario.
El comportamiento del gasto público se ve afectado por dos fuerzas contrarias, por un lado la caída en el empleo y la producción provoca que se reduzcan la base gravable y, por tanto, los ingresos fiscales, lo cual presiona para que el gasto público disminuya. Por otro lado, la menor deuda pública pasada implica que el gobierno tiene que destinar menos recursos para pagar esta. Por lo que, a priori, no es evidente que fuerza dominará. Este razonamiento se desprende de la ecuación (37).

$$
\begin{aligned}
& \frac{d g_{t+1}^{r}}{d \lambda_{t}}=\alpha\left(\frac{\lambda_{t+1}}{1-\lambda_{t+1}}\right)\left(\left(\beta \frac{y_{t+1}}{l_{t+1}}-\frac{\gamma}{1-\gamma} w_{t+1}\right) \frac{d l_{t+1}}{d \lambda_{t}}+\right. \\
& \left.\gamma \frac{y_{t+1}}{k_{t+1}} \frac{d k_{t+1}}{d \lambda_{t}}\right)-\frac{d\left(1+r_{t+1}\right)}{d \lambda_{t}} b_{t}-\left(1+r_{t+1}\right) \frac{d b_{t}}{d \lambda_{t}}
\end{aligned}
$$

En la ecuación (37), el primer miembro es la reducción en el ingreso tributario, el segundo es el aumento en el costo de la deuda pasada y el último es la disminución en la deuda pasada.

En los períodos subsecuentes a $t+1$, los precios y las asignaciones se comportarán de forma análoga a como lo hicieron en este período. Sin embargo, es importante resaltar que, a diferencia de lo que ocurre en este periodo, en los períodos posteriores la trayectoria que sigue el gasto público no es ambigua. El gasto público decrece de manera sistemática en los períodos subsecuentes, debido a la reducción en la masa tributaria y el aumento en el costo de la deuda. Por lo que, el incremento en el impuestos obliga al gobierno a reducir su gasto público de forma continua en los demás períodos, es decir, el gobierno se ve obligado a sostener una política fiscal restrictiva en el largo plazo con el fin de no incrementar su deuda. 


\subsection{Recesión y deuda pública}

En una economía en recesión y con altas tasas de desempleo el gobierno enfrenta presiones políticas para no seguir reduciendo su gasto público, aún si para hacerlo tiene que recurrir a incrementar su deuda. Se asumirá que en el período $t+2$ el gobierno decide no reducir su gasto público, pese a que sus ingresos tributarios disminuyeron y el costo de su deuda aumentó, para poder mantener su gasto el gobierno tendrá que recurrir a nueva deuda.

Con la finalidad de hacer viable este escenario se relajará la hipótesis sobre la temporalidad con la que el gobierno contrae deuda para financiar su gasto (véase hipótesis de sucesión de eventos). Se supondrá que el gobierno puede contraer deuda tanto al principio como al final del período para financiar su gasto del período, es decir, puede contratar deuda tanto en $a_{t+j}$ como en $a_{t+j+1}$ para financiar su gasto del período $t+j$, el cual ejerce en el momento $a_{t+j+1}$.

La deuda contratada al final del período se utilizará exclusivamente para garantizar que el gasto público no cambie, pese a la reducción de la masa tributaria y el mayor costo de la deuda pasada. Así en $\alpha_{t+3}$ el gobierno decide mantener su gasto fijo y financiarlo con mayor deuda. El hecho de que el gasto público no se reduzca, pese a la caída en los ingresos fiscales del gobierno, implica que la demanda efectiva disminuye en menor cuantía de lo que lo hizo el período pasado, esta se modifica en:

$\frac{d \hat{y}_{d, t+2}}{d \lambda_{t}}=\left(\frac{\alpha}{1+\lambda}\right) \gamma \frac{E\left(y_{t+2}\right)}{k_{t+2}} \frac{d k_{t+2}}{d \lambda_{t}}+\frac{d\left(1+r_{t+2}\right)}{d \lambda} b_{t+1}+\frac{d k_{t+3}}{d \lambda}$

El primer miembro de la ecuación (38) del lado derecho de la igualdad es la reducción del consumo debido a que la menor inversión pasada redujo el ingreso de los consumidores, el segundo miembro muestra que, al mantener constante su gasto el gobierno, el incremento en el costo de la deuda pública se traduce en un mayor consumo de los viejos. Adviértase que cuando el gasto público se ajustaba para garantizar que la deuda no creciera, el pago de la deuda pública no implicaba un mayor consumo de los acreedores del gobierno, debido a que el gobierno tenía que reducir su gasto en un monto equivalente al crecimiento del pago de su deuda. El último elemento refleja la disminución en la inversión presente.

La ecuación (38) muestra que la demanda se reducirá siempre que el efecto acumulado de la inversión sea superior al impacto positivo de mantener constante el gasto público, es decir:

$$
\left(\frac{\alpha}{1+\lambda}\right) \gamma \frac{E\left(y_{t+2}\right)}{k_{t+2}} \frac{d k_{t+2}}{d \lambda_{t}}+\frac{d k_{t+3}}{d \lambda} \lesseqgtr-\frac{d\left(1+r_{t+2}\right)}{d \lambda} b_{t+1} \rightarrow \frac{d \hat{y}_{d, t+2}}{d \lambda_{t}} \lesseqgtr 0
$$

Si bien es bastante plausible asumir que el efecto dominante sobre la demanda es la caída de la inversión, la ecuación nos muestra que no reducir el gasto público atenúa la caída de la demanda y en algunos casos puede incluso hacer crecer a esta.

Ante la caída en la inversión pasada, que redujo tanto la capacidad productiva como la demanda efectiva, el empleo se ajusta para garantizar que las empresas produzcan todo lo que el mercado les demanda. Por lo que, siempre que se adelante un período la ecuación (35), esta explicará cómo se comporta el nivel de empleo.

Sin embargo, debido a que al mantenerse constante el gasto público, la demanda se redujo en un menor monto que en el período pasado o incluso este pudo haber aumentado, es de esperarse que el empleo disminuya menos que en el periodo anterior o incluso pudo haber aumentado, dando signos de una recuperación de la economía. No obstante esta recuperación sería temporal debido a que la menor inversión y el mayor endeudamiento del gobierno provocarán que la inversión del período siguiente se reduzca, como se analizará un poco más adelante.

En la sección pasada se presentó que la evidencia estadística muestra que para España, Italia, Portugal, Irlanda y Grecia el empleo se comporta de la misma forma que la inversión, es decir, siempre que esta última se reduce, el empleo también lo hará. Con base en esta evidencia nosotros asumiremos que el esfuerzo por mantener el gasto público constante no fue suficiente para evitar la caída en la demanda efectiva y, por tanto, el empleo se ajustó a la 
baja aunque en una menor cuantía de lo que hubiera ocurrido si el gasto público se hubiera contraído.

¿Lo anterior contradice la idea de Krugman (2012) de que para salir de la crisis se requiere incrementar el gasto público? No. Simplemente muestra que mantener el gasto público constante no es suficiente para detener la caída de la economía, pero deja abierta la pregunta de cuánto tendría que incrementarse el gasto público para frenar la caída de la economía, esta pregunta no se abordará en este artículo, pero forma parte de la agenda de investigación.

La reducción en la demanda efectiva disminuyó la producción y, por tanto, contrajo los ingresos tributarios del gobierno, por otro lado el aumento del interés implica que creció el costo de la deuda pasada. Así el gobierno tendrá que incrementar su deuda para hacerle frente a sus obligaciones. La inecuación (39) nos muestra el comportamiento de la deuda pública en este período:

$$
\begin{aligned}
& \frac{d b_{f, t+2}}{d \lambda}=-\alpha\left(\frac{\lambda}{1+\lambda}\right)\left(\left(\beta \frac{y_{t+2}}{l_{t+2}}-\frac{\gamma}{1-\gamma} w_{t+2}\right) \frac{d t_{t+2}}{d \lambda}+\right. \\
& \left.\gamma \frac{y_{t+2}}{k_{t+2}} \frac{d k_{t+2}}{d \lambda}\right)+\frac{d\left(1+r_{t+2}\right)}{d \lambda} \frac{k_{t+2}}{\hat{y}_{d, t+2}}>0
\end{aligned}
$$

En la inecuación (39), el subíndice "f" en la deuda pública indica que esta se contrató al final del período. Esta inecuación nos muestra que el incremento en la deuda pública está explicado por tres elementos: i) la caída en el empleo, ii) la reducción en la inversión y iii) el alza en la tasa de interés. Los primeros dos reducen la base gravable del gobierno, el último incrementa el costo de la deuda pasada.

Para los períodos siguientes la economía se comporta de manera análoga a éste, aunque se agudiza la caída en la inversión, lo cual provoca que se requiera una mayor deuda para mantener el gasto público. Para analizar por qué es esto analicemos el período $t+3$.

Al inicio del período $t+3$, la tasa de interés aumenta debido a dos razones: i) el incremento en la deu- da pública $\left(\frac{d b_{f, t+2}}{d \lambda}\right)$, el cual aumentó la demanda de ahorro, ii) la menor inversión pasada, esta redujo la expectativa de ahorro.

La mayor tasa de interés reduce la inversión. Así, en el período $t+3$, la inversión disminuye en:

$$
\frac{d k_{t+4}}{d \lambda}=(1-\alpha) \gamma \frac{E\left(y_{t+3}\right)}{k_{t+3}} \frac{d k_{t+3}}{d \lambda}-\frac{d b_{f, t+2}}{d \lambda}<0
$$

Adviértase que la reducción en la inversión es mayor que en el período pasado (ecuación 33) la razón de esto es que la nueva deuda contratada por el gobierno $\left(\frac{d b_{f, t+2}}{d \lambda}\right)$ compite por recursos con la inversión. De manera análoga al período anterior la demanda efectiva se contrae debido a la caída en la inversión tanto presente como pasada, pese a que el gobierno mantiene su gasto constante. Por su parte, el empleo se ajusta para garantizar el equilibrio en el mercado de bienes.

El gobierno nuevamente se ve obligado a incrementar su deuda pública debido a dos razones i) la caída en la producción provoca que los ingresos fiscales se reduzcan, ii) la mayor tasa de interés incrementó el costo de la deuda pasada.

$$
\begin{aligned}
& \frac{d b_{f, t+3}}{d \lambda}=-\alpha\left(\frac{\lambda}{1+\lambda}\right)\left(\left(\beta \frac{y_{t+3}}{l_{t+3}}-\frac{\gamma}{1-\gamma} w_{t+3}\right) \frac{d t_{t+3}}{d \lambda}+\right. \\
& \left.\gamma \frac{y_{t+3}}{k_{t+3}} \frac{d k_{t+3}}{d \lambda}\right)+\frac{d\left(1+r_{t+3}\right)}{d \lambda} b_{t+2}+\left(1+r_{t+3}\right) \frac{d b_{f, t+2}}{d \lambda}
\end{aligned}
$$

La ecuación (40) muestra que la deuda pública se va incrementando. Adviértase que entre más grande es la caída en la inversión mayor es la deuda pública, por otro lado, (de acuerdo con la ecuación 39) entre más aumenta la deuda pública mayor será la caída de la inversión. Por lo que es un círculo vicioso, lo cual explica porque el crecimiento de la deuda va acompañado con una caída en la inversión y, por tanto, con menor producción.

En el siguiente apartado se analiza el vínculo entre la deuda pública y la inversión privada para cinco países de Europa para analizar si la propuesta teórica ofrecida es o no congruente con la evidencia estadística. 


\section{Evidencia empírica}

En este apartado se estudia el comportamiento de la deuda pública en cinco países de Europa, con la finalidad de analizar si la evidencia estadística es congruente con la explicación teórica que ofrece este modelo. De acuerdo a la ecuación 40, la dinámica de la deuda depende de la variación en el empleo y la inversión de forma inversa, es decir, a medida que estas dos últimas disminuyen, la deuda pública se incrementará. En contraste, el interés y la deuda pasada tienen una relación directa con la deuda pública presente. Para estimar estas relaciones se propone el siguiente modelo dinámico, donde se ocupa la misma nomenclatura que en el resto del documento:

$d b_{f, t}=a_{1}+a_{2} d b_{f, t-1}+a_{3} d k_{t}+a_{4} d t_{t}+a_{5} d r_{t}+e$

\subsection{Metodología}

Se utilizará el Método Generalizado de Momentos (MGM) en la versión de Arellano \& Bond (1988, 1991), la razón por la que se utiliza un modelo dinámico es porque en la propuesta teórica ofrecida, se muestra que la variable dependiente se emplea como un regresor, con un periodo rezagado. Arellano \& Bond $(1988,1991)$ desarrollan un estimador de variables instrumentales para corregir el problema de la correlación entre la primera diferencia del regresor $Y$ (rezagado un periodo) y la diferencia del término de error. De esta forma, se instrumenta con el regresor Y rezagado dos periodos. Cabe precisar que las estimaciones intra-grupos están sesgadas en modelos dinámicos, por lo que se tiene que aplicar el MGM, incluyendo como regresor a la variable dependiente rezagada, y que comúnmente se denomina variable instrumental.

Arellano \& Bover (1990) afirman que se pueden construir distintas variantes de estimadores MGM dependiendo de las propiedades que se supongan acerca de las variables explicativas que varían con el tiempo, incluyendo los casos en los que un subconjunto de estas son variables predeterminadas en lugar de estrictamente exógenas, pudiendo estar o no correlacionadas con los efectos.

Se estima un modelo dinámico de datos de panel, con observaciones anuales para las variables de estudio de los países: Grecia, España, Portugal, Irlanda e Italia, en el periodo 1990 a 2015 (Tabla 2).

Las estadísticas de deuda del gobierno se obtienen del Historical Public Debt Database (HPDD), del empleo en International Financial Statistics (IFS), ambas bases de datos del Fondo Monetario Internacional, la inversión de World Development Indicators del Banco Mundial y las tasas de interés del Banco Mundial y Bancos Centrales de los países estudiados.

Tabla 2. Datos utilizados para las variable

\begin{tabular}{|c|l|}
\hline Variable & \multicolumn{1}{|c|}{ Descripción } \\
\hline$d b_{t, t}$ & $\begin{array}{l}\text { Diferencia del logaritmo natural de la deuda del gobierno como porcentaje del PIB, a precios constantes (billones en moneda } \\
\text { local). }\end{array}$ \\
\hline$d b_{t, t-1}$ & $\begin{array}{l}\text { Diferencia del logaritmo natural de la deuda gubernamental como porcentaje del PIB, a precios constantes (billones en moneda } \\
\text { local) con un rezago. }\end{array}$ \\
\hline$d b_{t}$ & Diferencia del logaritmo natural de la inversión como porcentaje del PIB, a precios constantes (billones en moneda local). \\
\hline$d r_{t}$ & Diferencia de la tasa de interés sobre préstamos. \\
\hline$d t_{t}$ & Diferencia del logaritmo natural del empleo (en millones). \\
\hline
\end{tabular}

Fuente: Elaboración propia. 


\subsection{Resultados}

La estimación del modelo dinámico (descrito por la ecuación 41) indica que la deuda pública rezagada tiene un impacto positivo con la deuda presente (Tabla 3), cuyo coeficiente es 0.36 . La inversión y el empleo explican a la deuda pública presente en forma inversa, es decir, a medida que se incrementan estas dos variables exógenas la deuda pública disminuye. El coeficiente de la inversión es -0.31, del empleo es -0.20. Sin embargo, únicamente el estimador de la inversión es estadísticamente significativo. La tasa de interés modifica a la deuda en sentido positivo (0.001), aunque el coeficiente no es significativo en términos estadísticos.

La prueba de Sargan indica que no se puede rechazar la hipótesis nula, es decir, que las restricciones de sobre-identificación son válidas al 99 por ciento de confianza; y la prueba de Wald también arroja estimadores significativos a cualquier nivel de confianza.

Recordando, en el modelo teórico se plantea que la deuda pública presente y el interés tienen una dependencia directa con la deuda pública rezagada un periodo. Conforme a los resultados de la estimación, la relación se cumple tanto para la deuda pública, como para el interés (Tabla 3).

En contraste, el planteamiento teórico indica que a medida que el empleo y la inversión se incrementan, la deuda pública disminuirá; de acuerdo a los estimadores del modelo se puede concluir que se cumplen éstos efectos sobre la variable dependiente. Cabe precisar que el impacto que tiene la inversión en la deuda pública es mucho mayor que el del empleo. Empero, el incremento en la deuda pública resulta más explicado por el efecto mismo de la deuda pública rezagada en un periodo.

La evidencia estadística, para los países europeos en crisis económica, muestra que la disminución de la inversión privada aumentó la deuda pública. El modelo teórico aquí expuesto sugiere que la razón de esta relación estadística es que la inversión determina el comportamiento del ingreso nacional y, por tanto, de la recaudación, así a medida que la inversión disminuye también lo hará la recaudación del gobierno, forzándolo a incrementar la deuda pública si quiere mantener su gasto estable.
Tabla 3. Resultados de la estimación del modelo dinámico

\begin{tabular}{|c|c|}
\hline Variable dependiente: $\boldsymbol{d b}_{t, t}$ & Coeficiente \\
\hline$d b_{t,-1}$ & $\begin{array}{l}0.3684 \\
(0.000)\end{array}$ \\
\hline$d k_{t}$ & $\begin{array}{l}-0.3198 \\
(0.000) \\
\end{array}$ \\
\hline$d t_{t}$ & $\begin{array}{l}-0.2017 \\
(0.505) \\
\end{array}$ \\
\hline$d r_{t}$ & $\begin{array}{l}0.0013 \\
(0.746) \\
\end{array}$ \\
\hline$a_{1}$ & $\begin{array}{l}0.0302 \\
(0.000)\end{array}$ \\
\hline Prueba Wald & $\begin{array}{l}131.56 \\
(0.000)\end{array}$ \\
\hline Prueba de Sargan & $\begin{array}{l}185.82 \\
(0.7055)\end{array}$ \\
\hline No. Observaciones & 115 \\
\hline
\end{tabular}

Fuente: Elaboración propia.

Los valores entre paréntesis corresponden al $p$-value.

\section{Conclusiones}

En este artículo se mostró que un incremento en los impuestos destinado a pagar la deuda pública tiene inicialmente dos efectos: i) reduce la expectativa de ahorro (destruye recursos), ii) disminuye la deuda pública (libera recursos). Siempre que la expectativa de ahorro de los agentes se reduzca en un mayor monto que la deuda pública, la tasa de interés se incrementará, lo cual provocará que la inversión disminuya. Así, en un primer momento, el mayor impuesto reducirá el empleo, la producción, la inversión y la base gravable del gobierno. Sin embargo, pese a los costos en bienestar que un mayor impuesto implicó, la deuda pública se reducirá.

En los periodos posteriores al incremento en el impuesto, la caída en la inversión provocará que la economía se sitúe en una senda de decrecimiento, el desempleo involuntario aumentará siempre que la menor inversión reduzca a la demanda efectiva en un mayor monto que la capacidad productiva. El ingreso del gobierno se reducirá debido a que la caída en la producción reduce su base gravable, por otro lado, sus egresos aumentarán porque el 
incremento en la tasa de interés hace más costoso pagar su deuda. Por lo que, el gobierno se enfrenta a una disyuntiva: reducir el gasto público o contratar nueva deuda. Si decide la segunda opción, entonces se encontrará en una senda de caída en producción acompañada de incrementos en la deuda pública. Así, en el largo plazo, el incremento en los impuestos orientados a pagar la deuda pública tendrá un efecto contrario al deseado, pues éste en lugar de reducir la deuda, la incrementará. Es importante resaltar que en última instancia el efecto paradójico de los impuestos sobre la deuda pública se debe a que la inversión disminuye sistemáticamente.

La evidencia estadística para Grecia, Portugal, España, Irlanda e Italia muestra que la caída en la inversión en estos países explica el crecimiento de la deuda pública, por lo que las políticas de austeridad impuestas en estas sociedades no tendrán éxito en reducir la deuda pública mientras no crezca de forma sostenida la inversión. Más aún, del modelo teórico se infiere que las políticas de austeridad en lugar de reducir la deuda pública pueden hacerla crecer, pues fomenta la caída en la inversión.

\section{Referencias}

Alesina, A. \& Ardagna, S. (2010). Large Changes in Fiscal Policy: Taxes Versus Spending. Tax Policy and the Economy, 24.

Alesina, A. \& Perotti, R. (1995). Fiscal Expansions and Fiscal Adjustments in OECD Countries. Economic Policy, 10 (21), 205-248

Arellano, M., \& Bond, S. (1988). Dynamic Panel Data Estimation Using PPD: A Guide for Users. Institute for Fiscal Studies (IFS) Working Paper Series.

Arellano, M., \& Bond, S. (1991). Some Tests of Specification for Panel Data: Monte Carlo Evidence and an Application to Employment Equation. Review of Economic Studies, (58), 277-297.

Arellano, M., \& Bover, O. (1990). La Econometría de Datos de Panel. Investigaciones Económicas (Segunda Época) XIV (1), 3-45.

Argandoña, A., Gámez, C., \& Mochón, F. (1996) Macroeconomía Avanzada. I y II, España, Mc Graw Hill.

Barro, R. (1989). The Ricardian Approach to Budget Deficits. Journal of Economics Perspectives, 3 (2), 37-54.

Baxter, M., \& King, R. (1993). Fiscal Policy in General Equilibrium. American Economic Review, 83, 315-334

Benigno, P. (2015). New-Keynesian Economics: An AS-AD View. Research in Economics, 69 (4), 503-524.

Blanchard, O. \& Leigh, D. (2013). Growth Forecast Errors and Fiscal Multipliers, National Bureau of Economic Research, (w18779).
Clower, R. (1965). The Keynesian Counter-Revolution: A Theoretical Appraisal. En: Hahn, F. \& Brechling, F. (eds) The Theory of Interest Rate, Londres, Macmillan

Debreu, G. (1973). Teoría del Valor, España, Antoni Bosch.

Díaz, A. (2015). Crisis de la Zona Euro: Fallas Estructurales y Políticas de Austeridad. Economía UNAM, 13 (35), 45-68.

Guajardo, J., Leigh, D. \& Pescatori, A. (2014) Expansionary Austerity? International Evidence. Journal of the European Economic Association, 12 (4), 949-968.

Harrod, R. (1939). An Essay in Dynamic Theory. Economic Journal, 49. En: Sen, A. (1979). Lecturas: Economía del crecimiento, México, Fondo de Cultura Económica.

Heider, F. \& Ljungqvist, A. (2012). As certain as debt and taxes: Estimating the tax sensitivity of leverage from exogenous state tax changes. National Bureau of Economic Research, (w18263), 1-55.

Herndon, T., Ash, M. \& Pollin, R. (2014). Does High Public Debt Consistently Stifle Economic Growth? A Critique of Reinhart and Rogoff. Cambridge Journal of Economics, 38(2), 257-279.

Keynes, J. (1936). Teoría General de la Ocupación el Interés y el Dinero. México D.F. Fondo de Cultura Económica.

Krugman, P. (2012). Acabad ya con esta Crisis, Crítica, España.

Lasa, J. (1997). Deuda, Inflación y Déficit: Una Perspectiva Macroeconómica de la Política Fiscal, UAM, México D.F.

Linnemann, L., \& Schabert, A. (2003). Fiscal Policy in the New Neoclassical Synthesis. Journal of Money, Credit and Banking, 35 (6), 911-929.

Luna, V. \& Montes de Oca, R. (2015). Grecia: una Odisea Económica Reciente. Economía Informa, (392), 105-111.

Neffa, J., Féliz, M., Panigo, D. \& Pérez, P. (2006). Teorías Económicas Sobre el Mercado de Trabajo. 1 Marxistas y Keynesianos, Fondo de Cultura Económica, Argentina.

Noriega, F. (1994). Teoría del Desempleo, la Distribución y la Pobreza. México D.F. Ariel.

Noriega, F. (2001). Macroeconomía para el Desarrollo: Teoría de la Inexistencia del Mercado de Trabajo. México D.F. Mc Graw Hill.

Reinhart, C. \& Rogoff, K. (2010). Growth in a Time of Debt. American Economic Review, 100 (2), 573-578.

Robinson, J. (1980). Time in Economic Theory, Kyklos. 32(2), 219-229.

Rodríguez, A. (2005). Desempleo Involuntario en Equilibrio General Competitivo, Tesis doctoral, Universidad Autónoma Metropolitana.

Rodríguez, A. \& Venegas, F. (2015). Política fiscal europea y crisis económico-financiera actual. Contaduría y Administración, 60(S2), 54-82.

Stiglitz, J. (2010). Caída Libre: El Libre Mercado y El Hundimiento de la Economía Mundial, España, Taurus.

Velázquez, D. (2013a). Teoría de la Dinámica de las Economías de Mercado. Plaza y Valdés, México D.F.

Velázquez, D. (2013b). Un estudio comparativo sobre la formalización del axioma de racionalidad. Análisis Económico, XXVIII (68), 154-174.

Velázquez, D. (2014). ¿Qué efecto tiene un incremento en el impuesto al consumo, cuando éste se utiliza para reducir la deuda pública? Panorama económico, (17), 10-35.

Velázquez, D. (2015). El Efecto del Gasto Público en el Ciclo Económico: Una Visión Alternativa. Estudios Económicos, 30(1), 93-140. 


\section{ANEXO}

En este anexo se desarrolla la dinámica del modelo a partir de su recursividad. Sustituyendo la ecuación (7) en (8) y ésta en (21), y resolviendo para $l_{t}^{*}$ se obtiene:

$l_{t}^{*}=\frac{(1-\beta-\gamma)}{\beta}\left(\frac{\hat{y}_{d, t}}{k_{t}^{\gamma}}\right)^{\frac{1}{\beta}}$ (AI) Sustituyendo (AI) en (6) resulta: $l_{t}=\frac{(1-\gamma)}{\beta}\left(\frac{\hat{y}_{d, t}}{k_{t}^{\gamma}}\right)^{\frac{1}{\beta}}$ (AII) y $k_{t}=\left(\frac{\gamma}{1-\gamma}\right) \frac{w_{t} l_{t}}{\left(1+r_{t}\right)}$ (AIII). Sustituyendo (6) y (7) en 8 se obtiene: $y_{t}=\left(\frac{\beta}{1-\gamma} l_{t}\right)^{\beta} k_{t}^{\gamma}$ (AIV).

Sustituyendo (AIII) en la definición de ganancia y ésta y (AIV) en (9), (10) y (11) se obtiene: $c_{1, t}^{r}=\alpha\left(\frac{1}{1+\lambda_{t}}\right) H_{t}(\mathrm{AV})$, $c_{2, t+1}^{r}\left(1+\mathrm{r}_{\mathrm{t}+1}\right)(1-\alpha) \mathrm{H}_{\mathrm{t}}$ (AVI) y $s_{t}^{r}=(1-\alpha) \mathrm{H}_{\mathrm{t}}$ (AVII) Donde $\mathrm{H}_{t}=\left(\left(\frac{\beta}{1-\gamma} l_{t}\right)^{\beta} k_{t}^{\gamma}-\frac{\gamma}{1-\gamma} w_{t} l_{t}\right)$. Sustituyendo (AV) en la restricción presupuestal del gobierno, resulta: $g_{t}^{r}=\alpha\left(\frac{\lambda_{t}}{1+\lambda_{t}}\right) H_{t}+b_{t}-\left(1+r_{t}\right) b_{t-1}$ (AVIII) Sustituyendo (AV), (AVI) y (AVIII) en la definición de demanda efectiva se tiene: $\hat{y}_{d, t}=\alpha \mathrm{H}_{t}+\frac{\gamma}{1-\gamma} w_{t} l_{t}+k_{t+1}+b_{t}$ (AIX) Sustituyendo (AVII) y (AIII) en (14) se tiene: $\left(\frac{\gamma}{1-\gamma}\right) \frac{w_{t+1} l_{t+1}}{\left(1+r_{t+1}\right)}+b_{t}=(1-\alpha) \mathrm{H}_{t}(\mathrm{AX})$. Aplicando el operador de expectativas a (AVIII) $\mathrm{y}$ diferenciando con respecto a $b_{t} \mathrm{y} \lambda_{t}$ se obtiene: $\frac{d b_{t}}{d \lambda_{t}}=-\frac{\alpha}{\left(1-\lambda_{t}\right)^{2}} \mathrm{E}\left(\mathrm{H}_{t}\right)<0$ (AXI) Aplicando el operador E a (AIX) y diferenciando con respecto a $\mathrm{E}\left(\hat{y}_{d, t}\right)$ y $b_{t}$ y sustituyendo (AXI) resulta $\frac{d \mathrm{E}\left(\hat{y}_{d, t}\right)}{d \lambda_{t}}=\frac{d b_{t}}{d \lambda_{t}}<0$ (AXII) Obteniendo expectativas de (AV) y diferenciando con respecto a $\lambda_{t}$ y considerando (AXII) se tiene la ecuación (25).

Aplicando el operador $\mathrm{E}$ a (AII) y diferenciando con respecto a $\mathrm{E}\left(l_{t}\right)$ y $\lambda_{t}$, y considerando (25) se obtiene (26). Aplicado el operador de expectativas a $(\mathrm{AX})$ y diferenciando con respecto a $\left(1+r_{t+1}\right)$ y $\lambda_{t}$, y considerando que por (26) $\mathrm{E}\left(l_{t}\right)=$ $f\left(\lambda_{t}\right)$ se obtiene (22).

Diferenciando (AIII) con respecto a $k_{t} \mathrm{y} \lambda_{t}$, y considerando que por (22) $\left(1+r_{t+1}\right)=J\left(\lambda_{t}\right)$, Se obtiene (27). Diferenciando (AIX), y considerando que por (26) $\mathrm{E}\left(l_{t}\right)=f\left(\lambda_{t}\right)$ y por (27) $k_{t+1}=g\left(\lambda_{t}\right)$, se obtiene (28). Diferenciando (AII) con respecto a $l_{t}$ y $\lambda_{t}$, y considerando que por (28) $\hat{y}_{d, t}=\varphi\left(\lambda_{t}\right)$, se obtiene (29). Diferenciando (AIII) con respecto a $g_{t}^{r}$ y $\lambda_{t}$, y considerando que por (29) $l_{t}=\varphi\left(\lambda_{t}\right)$, se obtiene (30). Diferenciando A(X) con respecto a $\left(1+r_{t+2}\right)$ y $\lambda_{t}$, considerando que $k_{t+1}=g\left(\lambda_{t}\right)$ y E $\left(l_{t+1}\right) \neq f\left(\lambda_{t}\right)$, se obtiene (31). Diferenciando (AIII) con respecto a $k_{t+2} \mathrm{y} \lambda_{t}, \mathrm{y}$ considerando (31), se obtiene (33). Diferenciando (AIX) con respecto a $\hat{y}_{d, t+1}$ y $\lambda_{t}$, y considerando (33), se obtiene (34). Diferenciando (AI) con respecto a $l_{t+1}$ y $\lambda_{t}$, y considerando que por (27) $k_{t+1}=g\left(\lambda_{t}\right)$ y por (33) $\hat{y}_{d, t+1}=\Omega\left(\lambda_{t}\right)$ se tiene:

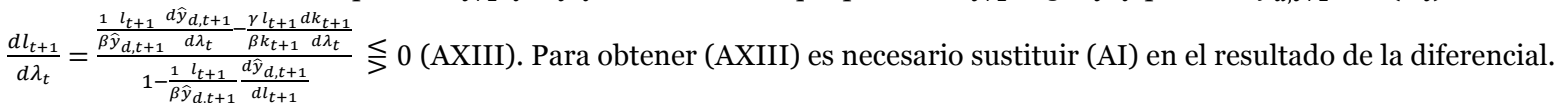
Asumiendo que el denominador de (AXIII) es positivo de su numerador resulta (35). Adviértase que por la función de producción $\gamma=\eta_{k, y}$.

El denominador positivo de la ecuación (AXIV) resulta siempre que $\beta>\frac{l_{t+1}}{\hat{y}_{d, t+1}} \frac{d \hat{y}_{d, t+1}}{d l_{t+1}}$ (AXV) Donde $\frac{l_{t+1}}{\hat{y}_{d, t+1}} \frac{d \hat{y}_{d, t+1}}{d l_{t+1}}=$ $\frac{l_{t+1}}{\hat{y}_{d, t+1}}\left(\alpha\left(\beta \frac{y_{t+1}}{l_{t+1}}-\frac{\gamma}{1-\gamma} w_{t+1}\right)\right)$ y $y_{t+1}=\left(\frac{\beta}{1-\gamma} l_{t+1}\right)^{\beta} k_{t+1}^{\gamma}(\mathrm{AXVI})$. La expresión (AXV) es verdadera si y sólo si (24) se verifica.

Diferenciando (AVIII) con respecto a $g_{t+1}^{r} y \lambda_{t}$ y considerando que $k_{t+1}, l_{t+1},\left(1+r_{t+1}\right)$ y $b_{t}$ están en función de $\lambda_{t}$ y la expresión (AXVI) se obtiene (36).

Partiendo de que $c_{2, t+1}^{r}=\left(1+r_{t+1}\right) s_{t}^{r}$ y sustituyendo (12) en esta expresión se tiene que $c_{2, t+1}^{r}=\frac{\gamma}{1-\gamma} w_{t+1} l_{t+1}+$ $\left(1+r_{t+1}\right) b_{t}$. Si la tasa de interés y el nivel de empleo no están en función de los impuestos resulta que $\frac{d c_{2, t+1}^{r}}{d \lambda_{t}}=(1+$ $\left.r_{t+1}\right) \frac{d b_{t}}{d \lambda_{t}}$, lo que valida que en el escenario (c) se afirma que $\frac{d g_{t+1}^{r}}{d \lambda_{t}}=-\frac{d c_{2, t+1}^{r}}{d \lambda_{t}}$. 